\title{
Genome-Wide Correlation of 36 Agronomic Traits in the 287 Pepper (Capsicum) Accessions Obtained from the SLAF-seq-Based GWAS
}

\author{
Lang Wu 1,2,†, Peng Wang 1,2,†, Yihao Wang 1,2, Qing Cheng 1,2, Qiaohua Lu 1,2, Jinqiu Liu 1,2, \\ Ting $\mathrm{Li}^{1,2}$, Yixin $\mathrm{Ai}^{1,2}$, Wencai Yang ${ }^{1,2}$, Liang Sun ${ }^{1,2, *}$ and Huolin Shen ${ }^{1,2, *}$ \\ 1 Beijing Key Laboratory of Growth and Developmental Regulation for Protected Vegetable Crops, \\ China Agricultural University, Beijing 100193, China; 15291832633@163.com (L.W.); \\ wp737@outlook.com (P.W.); yhwang0906@126.com (Y.W.); chengqing2013@126.com (Q.C.); \\ lqh12261842@163.com (Q.L.); 18811797502@163.com (J.L.); 15073196720@163.com (T.L.); \\ aiyixin0129@126.com (Y.A.); yangwencai@cau.edu.cn (W.Y.) \\ 2 Department of Vegetable Science, College of Horticulture, China Agricultural University, \\ No. 2 Yuanmingyuan Xi Lu, Haidian District, Beijing 100193, China \\ * Correspondence: liang_sun@cau.edu.cn (L.S.); shl1606@cau.edu.cn (H.S.); Tel.: +86-10-6273-1014 (L.S.); \\ +86-10-6273-2831 (H.S.) \\ + These authors contributed equally to this work.
}

Received: 5 October 2019; Accepted: 4 November 2019; Published: 13 November 2019

check for updates

\begin{abstract}
There are many agronomic traits of pepper (Capsicum L.) with abundant phenotypes that can benefit pepper growth. Using specific-locus amplified fragment sequencing (SLAF-seq), a genome-wide association study (GWAS) of 36 agronomic traits was carried out for 287 representative pepper accessions. To ensure the accuracy and reliability of the GWAS results, we analyzed the genetic diversity, distribution of labels (SLAF tags and single nucleotide polymorphisms (SNPs)) and population differentiation and determined the optimal statistical model. In our study, 1487 SNPs were highly significantly associated with 26 agronomic traits, and 2126 candidate genes were detected in the $100-\mathrm{kb}$ region up- and down-stream near these SNPs. Furthermore, 13 major association peaks were identified for 11 key agronomic traits. Then we examined the correlations among the 36 agronomic traits and analyzed SNP distribution and found 37 SNP polymerization regions (total size: $264.69 \mathrm{Mbp}$ ) that could be selected areas in pepper breeding. We found that the stronger the correlation between the two traits, the greater the possibility of them being in more than one polymerization region, suggesting that they may be linked or that one pleiotropic gene controls them. These results provide a theoretical foundation for future multi-trait pyramid breeding of pepper. Finally, we found that the GWAS signals were highly consistent with those from the nuclear restorer-of-fertility $(R f)$ gene for cytoplasmic male sterility (CMS), verifying their reliability. We further identified Capana06g002967 and Capana06g002969 as $R f$ candidate genes by functional annotation and expression analysis, which provided a reference for the study of cytoplasmic male sterility in Capsicum.
\end{abstract}

Keywords: pepper; agronomic traits; genome-wide association study (GWAS); specific-locus amplified fragment sequencing (SLAF-seq); multi-trait pyramid breeding; restorer-of-fertility $(R f)$ gene

\section{Introduction}

The agronomic traits of pepper (Capsicum L.) have many categories with abundant phenotypes, which affect the roots, stem, leaves, flowers, and fruits [1-3]. Agronomic traits of pepper are closely related to pepper growth, and previous correlation and path analyses have indicated that fruit color, shape, and weight can be directly used as economic indicators and consumer purchase criteria for 
pepper products [4]. Plant type (Pt), branching habit (Bh), leaf shape (Ls), leaf color (Lc), first flower node (Ffn), number of flowers per axil (Nfpa), flowering date, and percentage of fertile fruit also affected the yield of pepper [5-7]. Stem and leaf pubescence (Lp) and dry matter, capsaicinoid, vitamin $\mathrm{C}$, and anthocyanin content were correlated with resistance to disease and insect pests of pepper, which affected pepper production [8-12]. Therefore, mapping the genes that control agronomic traits is important for the development of the pepper industry (e.g., high-quality breeding and multi-trait pyramid breeding). However, most agronomic traits of pepper are controlled by quantitative trait loci (QTLs) and affected by environmental conditions, which makes it difficult to study these genes.

Previously, QTL mapping of agronomic traits in pepper has mainly focused on the agronomic traits closely associated with increasing pepper yield, such as fruit-related traits, disease resistance, and stress resistance (Table S1). For instance, using restriction fragment length polymorphism (RFLP), amplified fragment length polymorphism (AFLP), and random amplified polymorphic DNA (RAPD) markers, Ben Chaim et al., (2001) detected $f_{s} 3.1$ controlling fruit shape in an $\mathrm{F}_{3}$ population [13]. Blum et al. (2003) detected cap controlling capsaicin content on chromosome 7 in an $\mathrm{F}_{2}$ population using RAPD and sequence characterized amplified regions (SCARs) markers [14]. There have also been several reports on QTL analyses of other agronomic traits, such as $\mathrm{Pt}$, Bh, Ls, leaf margin (Lm), Ffn, and Nfpa [15-17]. However, there were no major QTLs detected, and the genes controlling QTLs have not been characterized.

In addition, some QTLs showed a cluster distribution in rice and in pepper [17,18], suggesting that these QTLs may be controlled either by one pleotropic gene or by a group of closely linked genes. Peterson (1959) found that the $A$ gene (controlling purple color) was linked to the $s w$ gene (controlling green color), and that both of them were linked to the $O$ gene (controlling oblate fruit shape) in pepper [19]. Ben Chaim et al. (2003) mapped two anthocyanin loci ( $F c$ and $A$ ) linked to a major quantitative trait locus, $f_{S}$ 10.1, for fruit-shape index [20]. These studies indicated that it is necessary to analyze the distribution of genes controlling the various traits of pepper, and then to explore the relationship between the traits at the genetic level.

The traditional QTL analysis, a gene mapping method, constructs separate populations (e.g., $\mathrm{BC}_{1}$, $\mathrm{F}_{2}, \mathrm{~F}_{3}$, recombinant inbred line, and double haploid) and then maps the genes based on mass molecular markers (e.g., RFLP, RAPD, AFLP, simple sequence repeat (SSR), cleaved amplified polymorphic sequence (CAPS), and insertion/deletion (INDEL)) (Table S1). Therefore, it is labor intensive, time consuming, and expensive, and the location accuracy is limited by the genetic diversity of the parents and the density of molecular markers used in the different populations [21-23]. With the rapid development of high-throughput sequencing technology, genome-wide association study (GWAS) based on linkage disequilibrium has become another powerful tool for analyzing complex agronomic traits [23]. This approach can overcome the limitations of QTL analysis and finds the association between single nucleotide polymorphisms (SNPs) and phenotype by detecting gene (locus) imbalances in natural populations [24]. This method has been successfully applied to the identification of candidate genes controlling complex agronomic traits in plant species, such as wheat, rice, maize, soya bean, chickpea, tomato, cabbage, cucumber, and alfalfa [25-32]. There have been few reports on the application of GWAS in pepper, and most experiments focused on only one or several agronomic traits. Through analyzing 96 pepper accessions by GWAS, Nimmakayala et al. (2014) found one marker on chromosome 1, which was significantly associated with capsaicin and dihydrocapsaicin content [33]. Two years later, Nimmakayala et al. (2016a, 2016b) examined the weight per fruit (Wpf) and fruit pedicel length (Fpl) by GWAS. Their results showed that there were 16 and 36 SNPs significantly associated with the Wpf and Fpl, respectively [34,35]. Ahn et al. (2018) also mapped the genes associated with powdery mildew resistance on chromosome 4 by GWAS [36].

GWAS can result in a high frequency of false positive errors for screening SNPs associated with traits [37]. Therefore, some researchers have combined traditional QTL mapping with GWAS to study the traits of arabidopsis, rice, maize, winter faba bean, and soybean [28,38-41]. Such combined approaches successfully avoid the limitations of applying the two methods separately and increase the 
reliability of the results. This combined strategy has also been successfully applied to pepper, with Han et al. (2018) identifying five candidate genes involved in capsaicinoid biosynthesis in the regions found by GWAS and QTL analysis [42]. However, the measures taken by these studies to improve the reliability of GWAS results were based on only one aspect (validation methods) and did not consider material population or statistical models.

For species with larger genomes, GWAS is also expensive. Specific-locus amplified fragment sequencing (SLAF-seq) is a strategy for discovering SNPs facilitated by reduced-representation genome sequencing and next-generation sequencing technologies. SLAF-seq is not only cheaper, but also obtains markers with high coverage and can be typed among populations, providing an efficient method for the detection of SNP markers in species with larger genomes, such as wheat, hulless barley, sunflower, and pepper. Three agronomic traits of pepper have been analyzed by SLAF-seq: Ffn, resistance to Phytophthora root rot, and resistance to Cucumber mosaic virus [43-45]. These studies demonstrated the efficiency of SLAF-seq as a strategy for the identification of SNPs (or genes) in pepper.

In our study, 36 agronomic traits were investigated in 287 pepper accessions in a GWAS (Table S2 and Note S1). The rates of false positive errors in GWAS analyses can be high owing to population structure and relatedness [22]. Therefore, the following analyses were carried out successively: (1) genetic diversity analysis to evaluate the phenotypic and genetic variation; (2) distribution analysis for SLAF tags and SNPs to assess the quality of development markers; (3) determination of optimal statistical model for each agronomic trait; (4) large-scale GWAS for the 36 agronomic traits; (5) construction of physical map based on the SNPs significantly associated with the 36 agronomic traits to explore correlations among agronomic traits; (6) verification of GWAS results based on fine mapping of male-sterility ( $R f$ gene) traits. Our objective was to identify candidate genes controlling agronomic traits important for pepper growing and explore the correlations among the 36 traits. Our findings provide key information for breeding high-quality pepper varieties and will be useful for developing multi-trait pyramid breeding strategies and increasing pepper yield.

\section{Results}

\subsection{Genetic Diversity Analysis for the GWAS Population}

In our study, 36 agronomic traits of pepper were surveyed and divided into 23 qualitative and 13 quantitative traits (Tables S3 and S4; Figures S1-S4). For the qualitative traits, 71 (80 in pepper germplasm resources) phenotypic types were identified, accounting for $88.75 \%$ of pepper germplasm resources (Table S3). The frequency distribution showed the main phenotype in those accessions (Figure S5). The coefficient of variation (CV) of 23 qualitative traits ranged from 0.09 to 0.74 . $\mathrm{Pt}$ and $\mathrm{Bh}$ had the lowest CV (0.09), and fruit shoulder shape (Fss) had the highest CV (0.74). The Shannon Wiener index $\left(\mathrm{H}^{\prime}\right)$ of 23 qualitative traits ranged from 0.14 (corolla color, $\mathrm{Cc}$ ) to 1.24 (fruit surface furrow, Fsf). For the 13 quantitative traits, the frequency of $\mathrm{Ffn}, \mathrm{Fpl}$, and fruit length $(\mathrm{Fl})$ were normally distributed (Figure S6). However, the frequency distribution of the other quantitative traits was positively skewed, indicating that a large proportion of the 287 pepper accessions had a lower value for these traits (Figure S6). In addition, mode, mean, min, max, range, $\mathrm{CV}$, and $\mathrm{H}^{\prime}$ were used to assess the variation in quantitative traits (Table S4). The CV of 13 quantitative traits ranged from 0.26 (Fpl) to 1.07 (Wpf). The $\mathrm{H}^{\prime}$ of 13 quantitative traits ranged from 0.45 (Nfpa) to 2.01 (Fl). Our results confirmed that most of the 36 agronomic traits had a rich phenotypic variation, suggesting that this population met the requirements for GWAS analysis.

\subsection{Identification and Distribution Analysis for Labels}

In total, $2238.81 \mathrm{M}$ paired-end reads were obtained from 287 pepper accessions based on HaeIII digestion. The average value of $\mathrm{Q}_{30}\left(\mathrm{Q}_{30}\right.$ indicates a quality score of 30 , indicating a $0.1 \%$ error rate or $99.9 \%$ sequence accuracy) and GC content were $95.72 \%(93.62-96.6 \%)$ and $39.30 \%(38.51-42.42 \%)$, respectively, indicating that our sequencing results for 287 pepper accessions were reliable (Table S5). 
Further analysis revealed that $1,824,874$ SLAF tags were obtained, with $287,910-475,273$ tags for each accession. The average sequencing depth of each accession was different (12.17-44.22; average, 19.62) but met the assumptions of the SLAF test (Table S6). Furthermore, 1,025,395 polymorphic SLAF tags were identified from 1,824,874 SLAF tags, and then a total of 9,557,790 SNPs were also developed from polymorphic SLAF tags (see Table S7 for SNP details).

To analyze the distribution of genetic information of SLAF tags and SNPs in the 287 accessions, we divided all accessions into 17 grades according to SLAF tag number, SNP number, SNP integrity, and SNP heterozygosity ratio (Table S8). In the population, both SLAF tags and SNPs were normally distributed (Figure S7). The eighth SLAF tag grade (SLAF tags ranged from 365,060 to 376,081) had the highest frequency (95) and percentage (33.10\%). The ninth SNP grade (SNPs ranged from 3,859,505 to $3,928,957)$ had the highest frequency (55) and percentage (19.16\%). SNP integrity was also normally distributed. These results showed that SLAF tags and SNPs were evenly distributed in the population, implying that their genetic information covered most of the accessions. However, the distribution of SNP heterozygosity ratio was positively skewed in the population, indicating that most of the accessions had a lower heterozygosity ratio.

As shown in Figure S8 and Figure 1, the distribution of SLAF tags and SNPs on chromosomes were also analyzed in our study. The percentage of polymorphic SLAF tags on every chromosome was over $50 \%$ of the total number of SLAF tags, and the percentage on chromosomes 5, 9, and 10 was high at $58.63 \%, 60.48 \%$, and $63.46 \%$, respectively. Chromosome 8 had the lowest percentage of polymorphic SLAF tags (53.13\%) (Figure S8). The density thermal map of SLAF tags and polymorphic SLAF tags had fewer color types and balanced distribution (Figure 1). Similar results were observed in the density heat map of SNPs, with only a small number of regions exhibiting high density. These results suggested that all SLAF tags, polymorphic SLAF tags, and SNPs were evenly distributed on every chromosome. This reduced the probability of missing important information on different chromosomes, suggesting that theses labels can represent the genetic information of the whole pepper genome and be used for GWAS analysis of the agronomic traits of pepper.

a
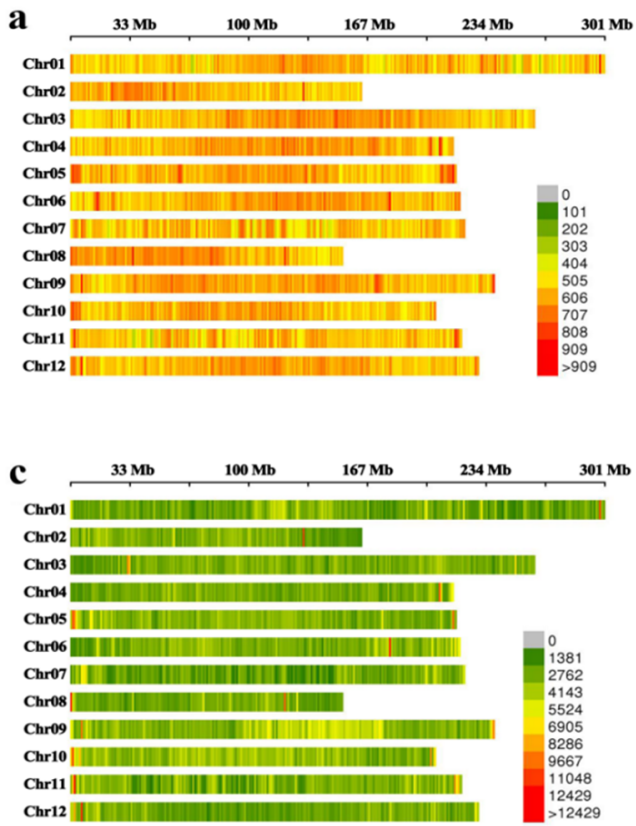

b

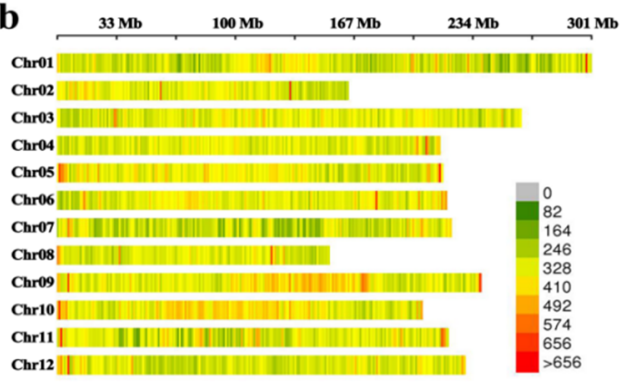

Figure 1. Density thermal map of specific-locus amplified fragment (SLAF), polymorphic SLAF (ploy-SLAF) and single nucleotide polymorphisms (SNPs) on chromosomes. (a) The number of SLAF within $1 \mathrm{Mb}$ window size. (b) The number of poly-SLAF within $1 \mathrm{Mb}$ window size. (c) The number of $\mathrm{SNPs}$ within $1 \mathrm{Mb}$ window size. Color index indicates the number of labels. 


\subsection{Population Structure Analysis for the GWAS Population}

In the present study, linkage disequilibrium was calculated for every chromosome using 594,429 SNPs (integrity $>0.5$; minor allele frequency $(\mathrm{MAF})>0.05$ ). Squared correlations of allele frequencies $\left(R^{2}\right)$ were used to investigate the extent of LD calculated within a 0-500-kb window. As shown in Figure S9, the LD decay distance for the 287 pepper accessions between all SNPs was $>500 \mathrm{~kb}$ when the value of the cut-off for $R^{2}$ was set at 0.1 , which showed that there was a greater probability of linkage among those SNPs in the accessions. In addition, the LD decays were not evenly distributed among chromosomes within the $0-500-\mathrm{kb}$ window. However, obvious LD differentiation was found among chromosome 1 and the other chromosomes.

Population structure and relatedness were the major factors leading to high rates of false positive errors in GWAS analysis [22]. Therefore, three methods were used to assess the population. First, a phylogenetic tree was constructed. As shown in Figure 2a, 287 pepper accessions were divided into four large categories and seven subcategories. Second, principal component analysis (PCA) was carried out. The interpretation rates of the first three principal components were $16.07 \%$ (PC1), 5.06\% (PC2), and 3.87\% (PC3), and cumulative variation was 25.01\% (Figure S10a). The PCA triplot showed that most accessions were scattered except for one subgroup (Figure 2b, Gif S1). Third, admixture software was used to calculate the clustering from $K=1-15$. As shown in Figure $S 10 b$, as the $K$ value increased, the cross validation error rate rapidly decreased, the first valley value appeared at $K=11$, the second valley value occurred at $K=13$, and then the cross validation error rate increased gradually as $K$ value increased. By comparing the value of cross validation error rate between $K=11$ and $K=13$, it was determined that $K=13$, which had a lower cross validation error rate, was the optimal subgroup number for this population. Detailed information of the subgroups for $K=13$ is shown in Figure $2 \mathrm{c}$ and Table S9. These results indicated a moderate level of population differentiation within different pepper subgroups.

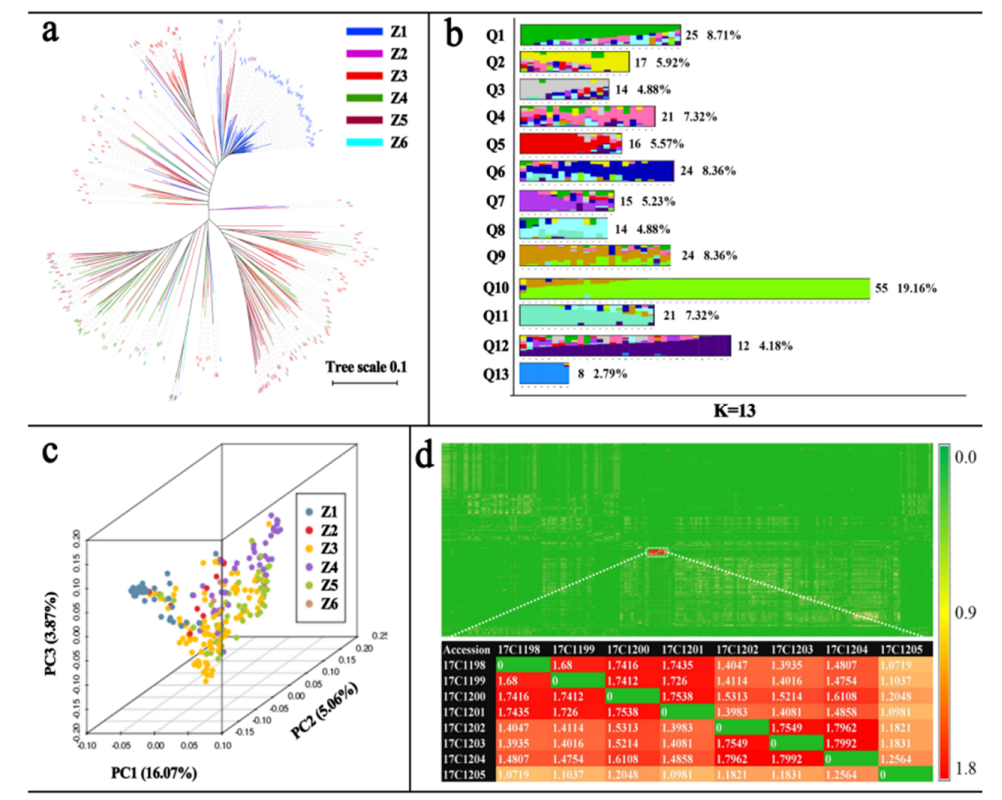

Figure 2. Population structure analysis for 287 pepper accessions. (a) NJ tree of 287 pepper accessions constructed from simple matching distance of genome SNPs. The Z1, Z2, Z3, Z4, Z5, and Z6 represent fruit shape is lantern, cons, horns (cattle and sheep horns), fingers, linear and the other, respectively. (b) Plots of the first three principal components of 287 pepper accessions. The Z1, Z2, Z3, Z4, Z5, and Z6 represent fruit shape is lantern, cons, horns (cattle and sheep horns), fingers, linear and the other, respectively. (c) Admixture in sub-populations $(K=13)$ resolved using 9,557,790 SNPs. The accessions were divided into 13 subgroups: Q1-Q13. The values represent accession number and percentage for each group. (d) Relatedness value thermal map of 287 pepper accessions. The color bar represents relatedness value. 
As shown in Figure S10c, the relatedness of most accessions $(76.44 \%)$ was $<0.1$ and was only $>0.4$ for $1.39 \%$ of accessions. The thermogram of relatedness also showed similar results with only eight accessions having a relatedness of $>1.05$ (Figure 2d). These results showed that most of the accessions were only distantly related, and thus, relatedness barely interfered with the GWAS analysis.

\subsection{Large-Scale GWAS for 36 Agronomic Traits}

The highly consistent filtered SNPs $(594,429)$ in the 287 pepper accessions were used for GWAS analysis of 36 agronomic traits. To reduce the influence of population structure and increase the reliability of GWAS results, we used five statistical models: general linear model (GLM), mixed linear model (MLM), compressed mixed linear model (CMLM), efficient mixed-model association expedited (EMMAX), and factored spectrally transformed linear mixed model (FaST-LMM). Performance of the five models was compared based on Q-Q plots (Figures S11-S14), and the most appropriate statistical model for each trait was selected for subsequent GWAS analysis. As shown in Table 1, Fast-LMM was the optimal model of 20 traits; EMMAX was the optimal model of 11 traits; GLM was the optimal model of five traits. These results showed the optimal model of different traits can be different, implying that it was necessary for each trait to select one optimal model for GWAS analysis.

Table 1. Details of peak regions associated with different traits identified via a genome wide association study (GWAS) in pepper.

\begin{tabular}{|c|c|c|c|c|c|c|c|}
\hline \multirow{2}{*}{ Plant Organ } & \multirow{2}{*}{ Trait } & \multirow{2}{*}{ Model } & \multirow{2}{*}{ Chromosome } & \multirow{2}{*}{$\begin{array}{c}\text { Number of SNPs }\left(p<1.707 \times 10^{-8}\right) \\
\text { in Peak Region }\end{array}$} & \multicolumn{3}{|c|}{ Trait-Associated Peak Region } \\
\hline & & & & & Start & End & Size (Mbp) \\
\hline \multirow[t]{21}{*}{ Stem } & \multirow[t]{9}{*}{$\mathrm{Pt}$} & \multirow[t]{9}{*}{ FaST-LMM } & Chr01 & 9 & 278425275 & 280417460 & 1.992185 \\
\hline & & & Chr04 & 2 & 23932585 & 23972683 & 0.040098 \\
\hline & & & Chr05 & 5 & 17744799 & 19221034 & 1.476235 \\
\hline & & & Chr06 & 10 & 5675663 & 5759294 & 0.083631 \\
\hline & & & Chr09 & 10 & 12510495 & 19373800 & 6.863305 \\
\hline & & & Chr09 & 14 & 118192571 & 119757960 & 1.565389 \\
\hline & & & Chr09 & 10 & 171029237 & 173134444 & 2.105207 \\
\hline & & & Chr11 & 3 & 37287718 & 40113909 & 2.826191 \\
\hline & & & Chr11 & 17 & 107259335 & 107501665 & 0.24233 \\
\hline & \multirow{8}{*}{$\mathrm{Bh}$} & \multirow{8}{*}{ EMMAX } & Chr01 & 59 & 275555035 & 277575138 & 2.020103 \\
\hline & & & Chr02 & 6 & 83643192 & 85422044 & 1.778852 \\
\hline & & & Chr02 & 21 & 104238462 & 104524382 & 0.28592 \\
\hline & & & Chr03 & 13 & 253213778 & 254639900 & 1.426122 \\
\hline & & & Chr06 & 14 & 13971060 & 18672334 & 4.701274 \\
\hline & & & Chr07 & 9 & 5373860 & 7707856 & 2.333996 \\
\hline & & & Chr07 & 35 & 184612786 & 184825374 & 0.212588 \\
\hline & & & Chr09 & 10 & 191839433 & 200002779 & 8.163346 \\
\hline & \multirow[t]{3}{*}{ Msc } & \multirow{3}{*}{ FaST-LMM } & Chr06 & 2 & 20231877 & 20231907 & 0.00003 \\
\hline & & & Chr08 & 6 & 114388151 & 114431559 & 0.043408 \\
\hline & & & Chr09 & 2 & 214330476 & 214330504 & 0.000028 \\
\hline & Msp & EMMAX & Chr11 & 28 & 27131483 & 28706358 & 1.574875 \\
\hline \multirow[t]{11}{*}{ Leaf } & Ls & GLM & - & - & - & - & - \\
\hline & Lc & GLM & - & - & - & - & - \\
\hline & \multirow[t]{5}{*}{$\mathrm{Lm}$} & \multirow[t]{5}{*}{ GLM } & Chr01 & 8 & 158420891 & 158729605 & 0.308714 \\
\hline & & & Chr04 & 7 & 58610574 & 59222198 & 0.611624 \\
\hline & & & Chr06 & 7 & 212371837 & 212424874 & 0.053037 \\
\hline & & & Chr08 & 7 & 131427437 & 134733324 & 3.305887 \\
\hline & & & Chr12 & 4 & 12354265 & 19260025 & 6.90576 \\
\hline & \multirow[t]{3}{*}{$\mathrm{Lp}$} & \multirow[t]{3}{*}{ EMMAX } & Chr10 & 9 & 162170912 & 162221229 & 0.050317 \\
\hline & & & Chr10 & 4 & 197927729 & 200867123 & 2.939394 \\
\hline & & & Chr12 & 3 & 22681366 & 22692847 & 0.011481 \\
\hline & Lsc & GLM & - & - & - & - & - \\
\hline \multirow[t]{13}{*}{ Flower } & \multirow[t]{9}{*}{$\mathrm{Cc}$} & \multirow[t]{9}{*}{ EMMAX } & Chr02 & 4 & 68060203 & 68060282 & 0.000079 \\
\hline & & & Chr04 & 6 & 189062369 & 192962061 & 3.899692 \\
\hline & & & Chr06 & 8 & 41630661 & 41857768 & 0.227107 \\
\hline & & & Chr07 & 2 & 214559213 & 214585881 & 0.026668 \\
\hline & & & Chr07 & 6 & 220688885 & 220941545 & 0.25266 \\
\hline & & & Chr08 & 8 & 135096972 & 135952487 & 0.855515 \\
\hline & & & Chr09 & 5 & 201471658 & 202362543 & 0.890885 \\
\hline & & & Chr11 & 5 & 28177723 & 28191389 & 0.013666 \\
\hline & & & Chr11 & 6 & 214289893 & 216304968 & 2.015075 \\
\hline & \multirow[t]{3}{*}{ Sc } & GLM & Chr01 & 3 & 211183454 & 211183884 & 0.00043 \\
\hline & & & Chr10 & 24 & 155035157 & 155097491 & 0.062334 \\
\hline & & & Chr11 & 114 & 27555236 & 29305711 & 1.750475 \\
\hline & Ac & FaST-LMM & Chr01 & 17 & 246812579 & 249477491 & 2.664912 \\
\hline
\end{tabular}


Table 1. Cont.

\begin{tabular}{|c|c|c|c|c|c|c|c|}
\hline \multirow{2}{*}{ Plant Organ } & \multirow{2}{*}{ Trait } & \multirow{2}{*}{ Model } & \multirow{2}{*}{ Chromosome } & \multirow{2}{*}{$\begin{array}{c}\text { Number of SNPs }\left(p<1.707 \times 10^{-8}\right) \\
\text { in Peak Region }\end{array}$} & \multicolumn{3}{|c|}{ Trait-Associated Peak Region } \\
\hline & & & & & Start & End & Size (Mbp) \\
\hline \multirow{44}{*}{ Fruit } & \multirow{10}{*}{$\begin{array}{l}\text { Ffn } \\
\text { Nfpa }\end{array}$} & \multirow{10}{*}{$\begin{array}{l}\text { FaST-LMM } \\
\text { EMMAX }\end{array}$} & Chr07 & 2 & 130948617 & 130973590 & 0.024973 \\
\hline & & & Chr01 & 7 & 280054497 & 280417460 & 0.362963 \\
\hline & & & Chr04 & 3 & 57766474 & 58558409 & 0.791935 \\
\hline & & & Chr05 & 2 & 206654389 & 206654579 & 0.00019 \\
\hline & & & Chr07 & 3 & 185249295 & 185271463 & 0.022168 \\
\hline & & & Chr09 & 22 & 10463866 & 12820022 & 2.356156 \\
\hline & & & Chr09 & 12 & 118192571 & 119641888 & 1.449317 \\
\hline & & & Chr10 & 7 & 29037048 & 33183732 & 4.146684 \\
\hline & & & Chr11 & 5 & 209423789 & 210867712 & 1.443923 \\
\hline & & & Chr12 & 20 & 192018036 & 193789860 & 1.771824 \\
\hline & \multirow{2}{*}{$\begin{array}{c}\text { Fpgs } \\
\text { Ms } \\
\text { Aif }\end{array}$} & \multirow{2}{*}{$\begin{array}{l}\text { EMMAX } \\
\text { FaST-LMM } \\
\text { EMMAX }\end{array}$} & Chr09 & 5 & 167226310 & 167226390 & 0.00008 \\
\hline & & & Chr10 & 86 & 148782468 & 155625398 & 6.84293 \\
\hline & Fsf & EMMAX & - & - & - & - & - \\
\hline & \multirow[t]{3}{*}{$\mathrm{Fg}$} & \multirow[t]{3}{*}{ FaST-LMM } & Chr07 & 8 & 6280167 & 6685034 & 0.404867 \\
\hline & & & Chr09 & 3 & 39263 & 1201526 & 1.162263 \\
\hline & & & Chr12 & 8 & 180112718 & 186338088 & 6.22537 \\
\hline & Fsc & EMMAX & - & - & - & - & - \\
\hline & \multirow[t]{5}{*}{ Fss } & FaST-LMM & Chr01 & 3 & 202642462 & 203121398 & 0.478936 \\
\hline & & & Chr04 & 2 & 15454448 & 15454730 & 0.000282 \\
\hline & & & Chr09 & 4 & 94667918 & 94693832 & 0.025914 \\
\hline & & & Chr09 & 8 & 126292337 & 127360615 & 1.068278 \\
\hline & & & Chr11 & 9 & 30762829 & 49631336 & 18.868507 \\
\hline & Fas & FaST-LMM & - & - & - & - & - \\
\hline & $\mathrm{Fpl}$ & FaST-LMM & - & - & - & - & - \\
\hline & $\mathrm{Pw}$ & FaST-LMM & Chr08 & 4 & 133185108 & 133433188 & 0.24808 \\
\hline & & & Chr12 & & 203552366 & 206591342 & 3.038976 \\
\hline & $\mathrm{Pl}$ & FaST-LMM & - & - & - & - & - \\
\hline & Psi & FaST-LMM & - & - & - & - & - \\
\hline & $\mathrm{FW}$ & FaST-LMM & Chr01 & 4 & 103860245 & 105083614 & 1.223369 \\
\hline & & & Chr08 & 22 & 133045048 & 133584887 & 0.539839 \\
\hline & & & Chr12 & 9 & 201671262 & 206591342 & 4.92008 \\
\hline & $\mathrm{Fl}$ & FaST-LMM & - & - & - & - & - \\
\hline & Fsi & EMMAX & - & - & - & - & - \\
\hline & Wpf & FaST-LMM & Chr01 & 7 & 103860245 & 105083614 & 1.223369 \\
\hline & & & Chr05 & 2 & 168537584 & 168540175 & 0.002591 \\
\hline & & & Chr08 & 4 & 133396929 & 133433188 & 0.036259 \\
\hline & & & Chr12 & 14 & 200798023 & 211814051 & 11.016028 \\
\hline & Dmc & FaST-LMM & Chr02 & 7 & 69956806 & 79414868 & 9.458062 \\
\hline & & & Chr11 & 5 & 85204434 & 85204791 & 0.000357 \\
\hline & $\mathrm{Tf}$ & FaST-LMM & Chr01 & 3 & 103860245 & 105083388 & 1.223143 \\
\hline & & & Chr08 & 3 & 126042291 & 133396929 & 7.354638 \\
\hline & & & Chr11 & 5 & 128979925 & 128979983 & 0.000058 \\
\hline & & & Chr12 & 8 & 200798023 & 207472045 & 6.674022 \\
\hline & Nol & FaST-LMM & - & - & - & - & - \\
\hline
\end{tabular}

Note: Pt, plant type; Bh, branching habit; Msc, main stem color; Msp, main stem pubescence; Ls, leaf shape; Lc, leaf color; Lm, leaf margin; Lp, leaf pubescence; Lsc, leaf surface characteristics; Cc, corolla color; Sc, style color; Ac, anther color; Ffn, first flower node; Nfpa, number of flowers per axil; Fpgs, flower pedicel growing state; Ms, Male-sterility; Aif, anthocyanin on immature fruit; Fsf, fruit surface furrow; Fg, fruit glossy; Fsc, fruit surface characteristics; Fss, fruit shoulder shape; Fas, fruit apex shape; Ab, appendage at blossom-end; Mfc, mature fruit color; St, spicy type; Fpl, fruit pedicel length; Pw, placenta width; Pl, placenta length; Psi, placenta size index; Fw, Fruit width; Fl, fruit length; Fsi, fruit shape index; Wpf, weight per fruit; Dmc, dry matter content; Tf, thickness of flesh; Nol, Number of locules (full text identical). Bold font represents the 13 quantitative traits classified by data type. '-', which indicated that there were no significant peaks.

In total, 1487 SNPs were identified with $p<1.707 \times 10^{-8}$ as the highly significant threshold from the optimal models, and 2126 candidate genes in the $100-\mathrm{kb}$ region up- or down-stream near those 
SNPs were detected for 26 traits (Table S10) [46,47]. The number of highly significant associated SNPs and genes for each trait and chromosome varied (Table S11). In addition, 247 associated peaks (SNPs were arranged neatly with a columnar distribution, Figures S15-S20) were identified for the 36 traits, and the 109 associated peaks above the highly significant threshold (blue lines in Manhattan plots) were defined as significant peaks (strong association signals) for 26 traits. According to the number and physical location of SNPs in the significant peaks, 91 significant peak regions were identified for 22 traits (Table 1). The important SNPs and genes in the significant peak regions were highlighted in red (Table S10), and the important genes were annotated (Table S12).

\subsubsection{The Three Stem-Related Traits}

\section{Plant Type}

In total, 104 SNPs (184 genes) mainly distributed on chromosomes 1-9, 11, and 12 were significantly associated with Pt (Table S11). Chr11_37287718, Chr04_214680400, and Chr09_169528955 were the most highly associated with Pt (Table S10). Nineteen SNP polymerization regions were highlighted on chromosomes 1, 3, 6, 7, 8, 9, 11, and 12, in which the SNP associated with Pt and the SNP associated with the other traits had polymerization (Figure 3). Specifically, Pt and Bh, Pt and Cc, and Pt and Nfpa had 15, 16, and 13 polymerization regions, respectively (Table S17). Moreover, 10 highly significant peaks associated with $\mathrm{Pt}$ were observed on chromosomes 1, 5-7, 9, and 11, with no main peak evident (Figure S15). Furthermore, nine significant peak regions (total size: $17.19 \mathrm{Mbp}$ ), distributed on chromosomes 1, 4, 5, 6, 9, and 11 were identified for Pt (Table 1).

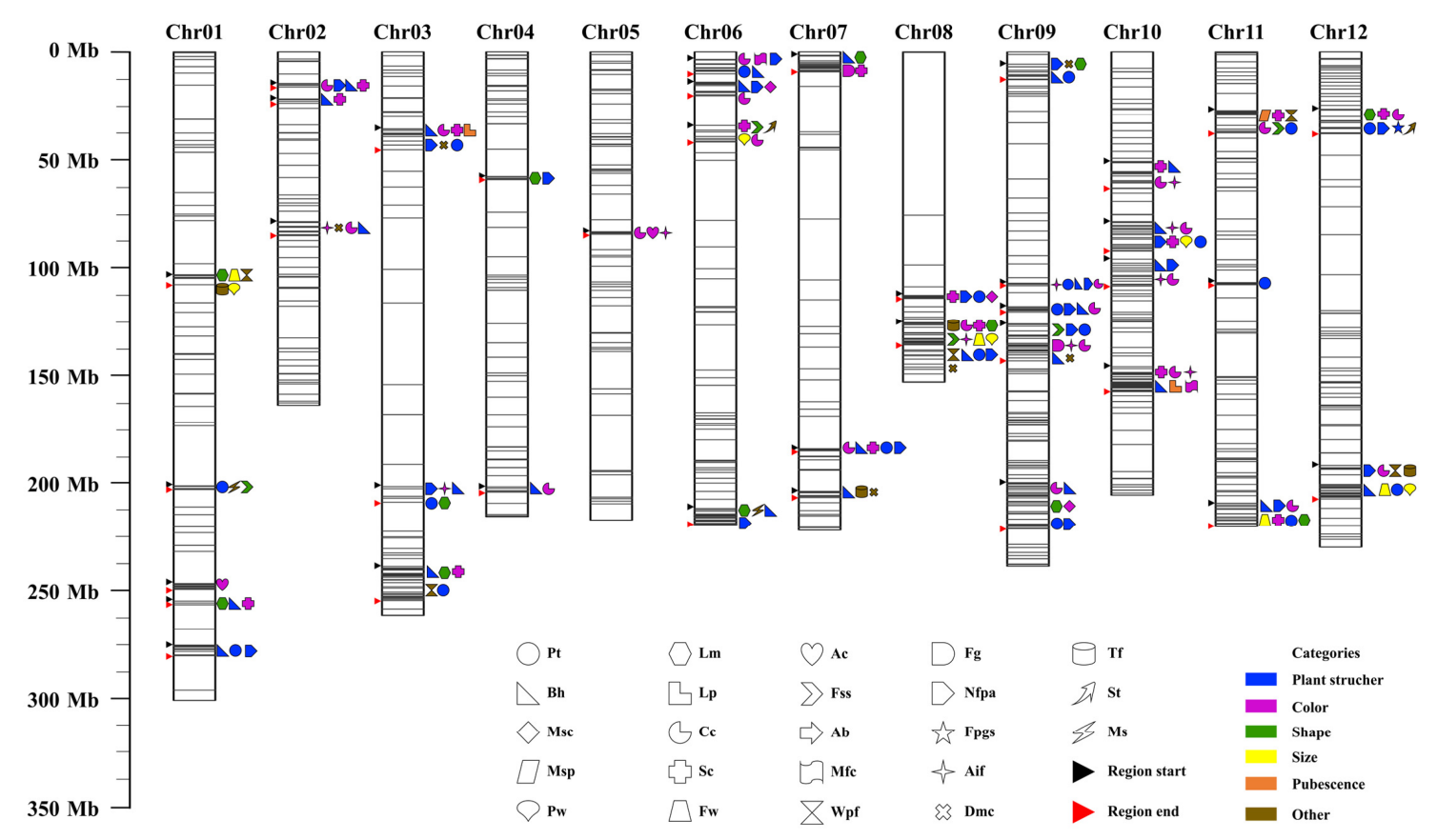

Figure 3. The position of 37 polymerization region for 23 agronomic traits on each chromosome. On the left aside of the chromosomes, the starting position of polymerization region was indicated by the black triangle, and the end position of that was indicated by the red triangle. Different colors and shapes represent the trait in various categories. 


\section{Branching Habit}

In total, 307 SNPs (548 genes) distributed on all chromosomes were significantly associated with Bh (Table S11). Chr10_51277133, Chr03_240589834, and Chr10_99038533 were the most highly associated with Bh (Table S10). Then, 27 SNP polymerization regions were highlighted on chromosomes 1-4 and 6-12, in which the SNP associated with Bh and the SNP associated with the other traits had polymerization (Figure 3). Specifically, Bh and Lm, Bh and Cc, Bh and style color (Sc), Bh and Nfpa, and Bh and Aif had 9, 18, 12, 18, and 9 polymerization regions, respectively (Table S17). Moreover, eight highly significant peaks associated with Bh were observed on chromosomes 1-3, 6, 7, and 9, with no main peak evident (Figure S15). Furthermore, eight significant peak regions (total size: $20.92 \mathrm{Mbp}$ ), distributed on chromosomes 1, 2, 3, 6, 7, and 9, were identified for Bh (Table 1).

\section{Main Stem Pubescence}

In total, 22 SNPs (two genes) mainly distributed on chromosome 11 were significantly associated with main stem pubescence (Msp) (Table S11). Chr11_27217603, Chr11_27217695, and Chr11_27217636 were the most highly associated with Msp (Table S10). One SNP polymerization region was highlighted on chromosome 11, in which the SNP associated with Msp and the SNP associated with the other traits (Cc, Sc, Fss, and Wpf) had polymerization (Figure 3). Moreover, one highly significant peak on chromosome 11 was the main peak associated with Msp (Figure S19). Furthermore, one significant peak region (size: $1.57 \mathrm{Mbp}$ ), located on chromosome 11, was identified for Msp (Table 1).

\subsubsection{The Two Leaf-Related Traits}

\section{Leaf Margin}

In total, 117 SNPs (247 genes) mainly distributed on chromosomes 1 and 3-12 were significantly associated with Lm (Table S11). Chr09_213296577,CChr08_134733299, and Chr10_59043555 were the most highly associated with Lm (Table S10). Twelve SNP polymerization regions were highlighted on chromosomes 1, 3, 4, 6, 7, 8, 9, 11, and 12, in which the SNP associated with Lm and the SNP associated with the other traits had polymerization (Figure 3). Specifically, Lm and Cc, Lm and Sc, and Lm and Nfpa had 4, 6, and 8 polymerization regions, respectively (Table S17). Moreover, five highly significant peaks were observed on chromosomes 1, 4, 6, 8, and 12, with no main peak evident (Figure S17). Furthermore, five significant peak regions (total size: $11.18 \mathrm{Mbp}$ ), distributed on chromosomes 1, 4, 6, 8 , and 12 were identified for Lm (Table 1).

\section{Leaf Pubescence}

In total, $17 \mathrm{SNPs}$ (51 genes) mainly distributed on chromosomes 2, 3, 9, 10, and 12 were significantly associated with Lp (Table S11). Chr10_154508628, Chr09_178710956, and Chr10_197927729 were the most highly associated with Lp (Table S10). Two SNP polymerization regions were highlighted on chromosomes 3 and 10, in which the SNP associated with Lp and the SNP associated with the other traits had polymerization (Figure 3). Specifically, Lp and Cc, and Lp and Sc had two polymerization regions, respectively (Table S17). Moreover, three highly significant peaks were observed on chromosomes 10 and 12, with the former being the main peak associated with Lp (Figure S19). Furthermore, three significant peak regions (total size: $3.00 \mathrm{Mbp}$ ), distributed on chromosomes 10 and 12, were identified for Lp (Table 1). 


\subsubsection{The Five Flower-Related Traits}

\section{Corolla Color}

In total, 125 SNPs (279 genes) distributed on all of chromosomes were significantly associated with Cc (Table S11). Chr02_85429287, Chr09_201616976, and Chr08_138467669 were the most highly associated with Cc (Table S10). Then, 22 SNP polymerization regions were highlighted on chromosomes 2-12, in which the SNP associated with Cc and the SNP associated with the other traits had polymerization (Figure 3). Specifically, Cc and Sc, Cc and Nfpa, and Cc and Aif had 11, 15, and 9 polymerization regions, respectively (Table S17). Moreover, eight highly significant peaks were observed on chromosomes 2, 4, 6-9, and 11, with no main peak evident (Figure S16). Furthermore, nine significant peak regions (total size: $8.18 \mathrm{Mbp}$ ), distributed on chromosomes 2, 4, 6, 7, 8, 9 and 11, were identified for Cc (Table 1).

\section{Style Color}

In total, 291 SNPs (152 genes) distributed on all of chromosomes were significantly associated with Sc (Table S11). Chr10_157424507, Chr10_155327941, and Chr10_155327958 were the most highly associated with Sc (Table S10). Sixteen SNP polymerization regions were highlighted on chromosomes $1,2,3,6,7,8,10,11$, and 12, in which the SNP associated with Sc and the SNP associated with the other traits had polymerization (Figure 3). Specifically, Sc and Nfpa, Sc and Aif had 8 and 4 polymerization regions, respectively (Table S17). Moreover, six highly significant peaks were observed on chromosomes $1-3,8,10$, and 11, with the latter two being the main peaks associated with Sc (Figure S16). Furthermore, three significant peak regions (total size: $1.81 \mathrm{Mbp}$ ), distributed on chromosomes 1, 10, and 11, were identified for Sc (Table 1).

Number of Flowers Per Axil

In total, 126 SNPs (221 genes) distributed on all of chromosomes were significantly associated with Nfpa (Table S11). Chr12_193629045, Chr12_193629029, and Chr12_193628684 were the most highly associated with Nfpa (Table S10). Then, 21 SNP polymerization regions were highlighted on chromosomes 1-4 and 6-12, in which the SNP associated with Nfpa and the SNP associated with the other traits had polymerization (Figure 3). Specifically, Nfpa and Aif, Nfpa and dry matter content (Dmc) had 6 and 4 polymerization regions, respectively (Table S17). Moreover, 11 highly significant peaks were observed on chromosomes 1, 4-7, 9-10, and 12, with the latter being the main peak associated with Nfpa (Figure S15). Furthermore, 12 significant peak regions (total size: $18.48 \mathrm{Mbp}$ ), distributed on chromosomes 1, 4, 5, 6, 7, 9, 10,11, and 12, were identified for Nfpa (Table 1).

\section{Flower Pedicel Growing State}

In total, 16 SNPs (14 genes) distributed on chromosome 12 were significantly associated with flower pedicel growing state (Fpgs) (Table S11). Chr12_37932067, Chr12_37994286, and Chr12_37994288 were the most highly associated with Fpgs (Table S10). One SNP polymerization region was highlighted on chromosome 12, in which the SNP associated with Fpgs and the SNP associated with the other traits (Lm, Sc, Cc, Pt, Nfpa, and spicy type (St)) had polymerization (Figure 3). Moreover, one highly significant peak on chromosome 12 was the main peak associated with Fpgs (Figure S15). Furthermore, one significant peak region (size: $5.14 \mathrm{Mbp}$ ), located on chromosome 12, was identified for Fpgs (Table 1). 
Male Sterility

In total, 78 SNPs (17 genes) mainly distributed on chromosomes 1, 6, 10, and 11 were significantly associated with male sterility (Ms) (Table S11). Chr06_215261283, Chr06_215468191, and Chr06_215411595 were the most highly associated with Ms (Table S10). Two SNP polymerization regions were highlighted on chromosomes 1 and 6, in which the SNP associated with Ms and the SNP associated with the other traits had polymerization (Figure 3). Moreover, one highly significant peak on chromosome 6 was the main peak associated with Ms (Figure S20). Furthermore, one significant peak region (size: $1.09 \mathrm{Mbp}$ ), located on chromosome 6, was identified for Ms (Table 1).

\subsubsection{The Five Fruit-Related Traits}

Anthocyanin on Immature Fruit

In total, 102 SNPs (96 genes), mainly distributed on chromosomes 1-5 and 7-10, were significantly associated with anthocyanin on immature fruit (Aif) (Table S11). Chr07_105839253, Chr10_75362680, and Chr10_107762896 were the most highly associated with Aif (Table S10). Ten SNP polymerization regions were highlighted on chromosomes 2, 3, 5, 9, and 10, in which the SNP associated with Aif and the SNP associated with the other traits had polymerization (Figure 3). Specifically, Aif and Fss, Aif and placenta width $(\mathrm{Pw})$, and Aif and Dmc had 2, 2, and 3 polymerization regions, respectively (Table S17). Moreover, seven highly significant peaks were observed on chromosomes 2, 4, and 7-10, with the latter being the main peak associated with Aif (Figure S16). Furthermore, four significant peak regions (total size: $7.59 \mathrm{Mbp}$ ), distributed on chromosomes 8, 9, and 10, were identified for Aif (Table 1).

\section{Mature Fruit Color}

In total, 16 SNPs (49 genes) distributed on chromosome 6 were significantly associated with mature fruit color (Mfc) (Table S11). Chr06_10060744,Chr06_8776355, and Chr06_8769243 were the most highly associated with Mfc (Table S10). Two SNP polymerization regions were highlighted on chromosomes 6 and 10, in which the SNP associated with Mfc and the SNP associated with the other traits had polymerization (Figure 3). Moreover, one highly significant peak on chromosome 6 was the main peak associated with Mfc (Figure S16). Furthermore, one significant peak region (size: $6.30 \mathrm{Mbp}$ ), located on chromosome 6, was identified for Mfc (Table 1).

\section{Spicy Type}

In total, 44 SNPs (29 genes) mainly distributed on chromosomes 3, 4, 9, 10, and 12 were significantly associated with St (Table S11). Chr12_35372851, Chr12_35623982, and Chr12_35624024 were the most highly associated with St (Table S10). Two SNP polymerization regions were highlighted on chromosomes 6 and 12, in which the SNP associated with St and the SNP associated with the other traits had polymerization (Figure 3). Moreover, six highly significant peaks were observed on chromosomes 3, 4, 9, 10, and 12, with the latter being the main peak associated with St (Figure S20). Furthermore, six significant peak regions (total size: $8.22 \mathrm{Mbp}$ ), distributed on chromosomes 4, 5, 6, 10, and 12, were identified for St (Table 1). 
Placenta Width

In total, seven SNPs (18 genes) distributed on chromosomes 1, 6, 8, and 12 were significantly associated with Pw (Table S11). Chr12_206591342,CChr06_40911519, and Chr08_133396929 were the most highly associated with Pw (Table S10). Five SNP polymerization regions were highlighted on chromosomes 1, 6, 8, 10 and 12, in which the SNP associated with Pw and the SNP associated with the other traits had polymerization (Figure 3). Specifically, Pw and fruit width (Fw), Pw and Wpf, and Pw and thickness of flesh (Tf) each had three polymerization regions (Table S17). Moreover, four highly significant peaks were observed on chromosomes 1, 8, and 12, and two of them (on chromosomes 8 and 12) were the main peaks associated with Pw (Figure S18). Furthermore, two significant peak regions (total size: $3.27 \mathrm{Mbp}$ ), distributed on chromosomes 8 and 12, were identified for Pw (Table 1).

Fruit Width

In total, 26 SNPs (65 genes) distributed on chromosomes 1, 4, 8, and 12 were significantly associated with Fw(Table S11). Chr08_133396929, Chr08_133433188, and Chr08_133185108 were the most highly associated with Fw (Table S10). Four SNP polymerization regions were highlighted on chromosomes 1, 8, 11, and 12, in which the SNP associated with Fw and the SNP associated with the other traits had polymerization (Figure 3). Specifically, Fw and Wpf, and Fw and Tf each had three polymerization regions (Table S17). Moreover, four highly significant peaks were observed on chromosomes 1, 4, 8, and 12, with the peak on chromosome 8 being the main peak associated with Fw (Figure S18). Furthermore, three significant peak regions (total size: 6.68 Mbp), distributed on chromosomes 1, 8, and 12, were identified for Fw (Table 1).

There were few to no SNPs significantly associated with the remaining 21 traits: main stem color (Msc), Ls, Lc, leaf surface characteristics (Lsc), Ffn, anther color (Ac), fruit glossy (Fg), Fsf, fruit surface characteristics (Fsc), Fss, appendage at blossom-end ( $\mathrm{Ab}$ ), Fpl, placenta length (Pl), Fl, fruit apex shape (Fas), fruit shape index (Fsi), placenta size index (Psi), Wpf, Dmc, Tf, and number of locules (Nol). However, there were many peaks with relatively small effects observed distributed on multiple chromosomes (Figures S15-S18, S20). These findings implied that these traits may be controlled by multiple micro-genes.

\subsection{Global Exploration of Correlations Among the 36 Agronomic Traits}

We explored the correlations among the 36 traits using correlation network analysis (Figure 4). The strongest positive correlation was observed among the 11 fruit-related traits: Fpl, Pw, Pl, Psi, Fw, $\mathrm{Fl}, \mathrm{Fsi}, \mathrm{Nol}, \mathrm{Wpf}, \mathrm{Tf}$, and Dmc. For stem-related traits, Bh was negatively correlated with $\mathrm{Pt}(-0.49)$, and Msc was positively correlated with Msp (0.25) and Pt (0.23). Correlations among leaf-related traits and flower-related traits were less significant than those among stem-related traits. Correlation coefficients among pairwise traits are shown in Table S14.

We compared their shared peak numbers in Manhattan plots based on descriptive classifications (Figures S15-S20). For plant structure-related traits, $\mathrm{Pt}, \mathrm{Bh}$, and Nfpa had a total of three shared peaks distributed on chromosomes 1, 6, and 7. However, there was one shared peak on chromosome 2 for Bh and Fpgs, and one shared peak on chromosome 12 for Fpl and Fpgs.

For color-related traits, $\mathrm{Cc}$ and Msc, and $\mathrm{Cc}$ and Fg each had one shared peak distributed on chromosomes 9 and 8, respectively. Cc, Lc, and Fg had one shared peak on chromosome 12. Cc, Ac, and Aif had one shared peak on chromosome 2. Sc and Msc had one shared peak on chromosomes 8 and 10, respectively. Sc, Aif, and Mfc had one shared peak on chromosome 10. Ac and Aif had one shared peak on chromosomes 4 and 7, respectively. Msc and Mfc had one shared peaks on chromosomes 10. 


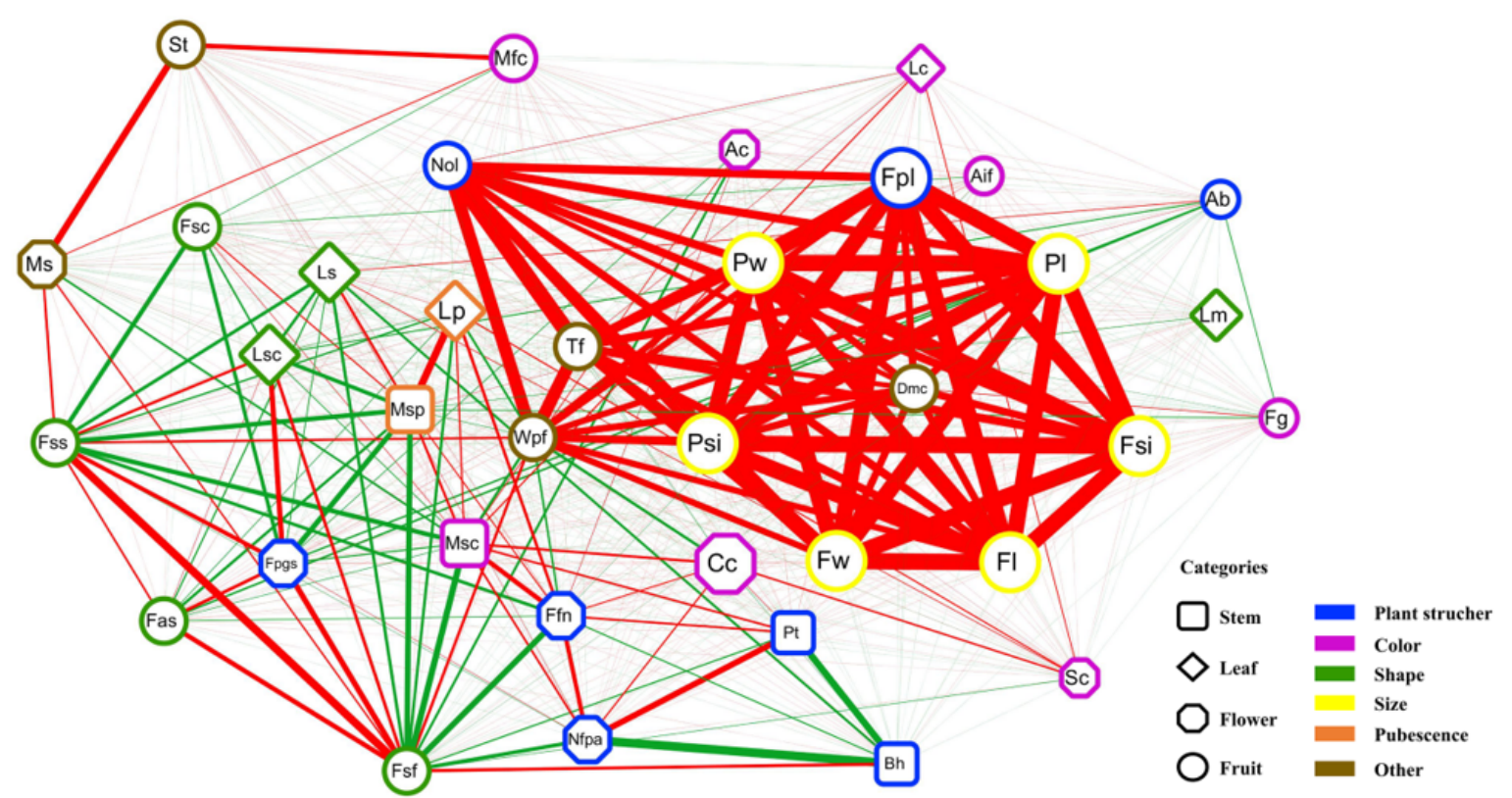

Figure 4. Phenotype correlation network of agronomic traits in pepper. Red and green lines represent negative and positive correlations, respectively. Line width is proportional to the strength of the correlation. Different shape nodes represent stem-related, leaf-related, flower-related and fruit-related traits, respectively. Different color frames of nodes represent plant structure-related, color-related, shape-related, size-related, pubescence-related and other traits, respectively.

For shape-related-traits, Ls and Lsc had one shared peak on chromosome 6. Ls and Fas had four shared peaks distributed on chromosomes 1, 2, 10, and 12. Moreover, Fsc and Fsf, and Fsc and Fas each had one shared peak on chromosomes 10 and 12, respectively.

For organ size-related traits, Fw and Pw had four shared peaks on chromosomes 1, 4, 8, and 12. Fl and Pl had two shared peaks distributed on chromosomes 6 and 8. Fsi and Psi had one shared peak on chromosomes 2 and 7, respectively.

For pubescence-related traits, Msp and Lp had one shared peak on chromosome 10, and it was the main peak of Lp. However, the main peak of Msp was on chromosome 11, suggesting that the major genes controlling the two traits may be different. There were more peaks for Lp (eight) than Msp (two), implying that Lp was controlled by minor genes.

For other traits, there were six shared peaks between $\mathrm{Wpf}$ and $\mathrm{Tf}$ on chromosomes 1, 3, 5, 8, 11, and 12. St and Tf had one shared peak on chromosomes 3 and 8, respectively. St and Dmc, and St and Ms each had one shared peak each on chromosomes 6 and 2, respectively. We compared the distribution of peaks of the traits between the different categories and observed one or two shared peaks for Sc and Fsf (chromosome 10), Sc and Fss (chromosomes 8 and 11), Fas and Fg (chromosome 12), and St and Fpgs (chromosome 12). It was worth noting that Sc, Fss, and Msp had one shared peak (chromosome 11), that Sc, Aif, and Lp had one shared peak (chromosome 10), and that Sc, Cc, Fw, and Pw had one shared peak (chromosome 08).

Additionally, we identified the significant SNPs near peaks for pairwise traits and summarized the SNP and gene numbers. As shown in Figure 5, we found that among the significant SNPs, some of them were associated with more than one trait. For instance, 46 SNPs (60 genes) were associated with Pt and Nfpa, and 10 SNPs (39 genes) were associated with Bh and Nfpa. Based on pairwise traits, 385 genes were associated with more than one trait, indicating gene pleiotropy. For detailed information, see Table S15. 


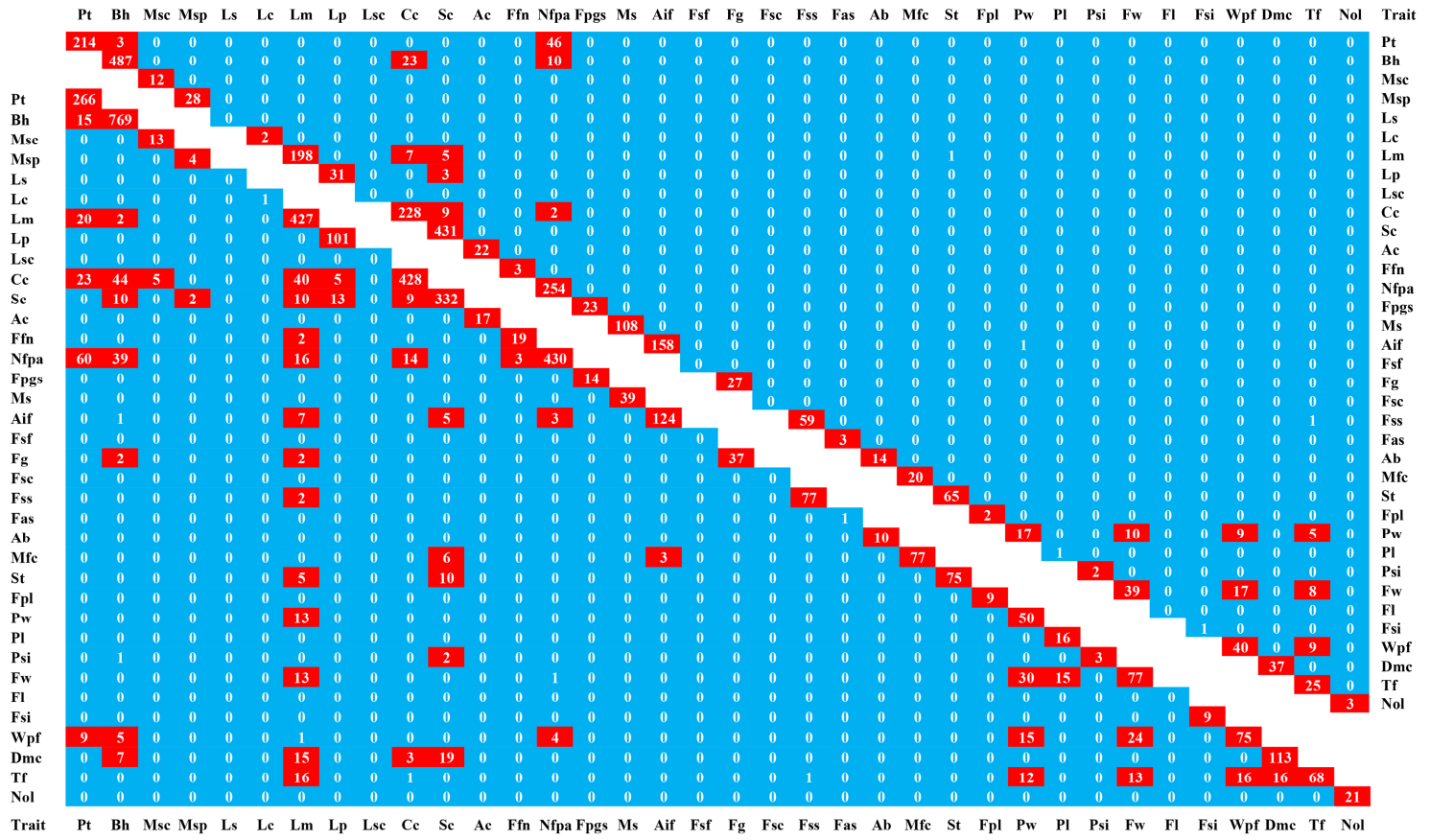

Figure 5. Significant SNPs and potential candidate gene numbers in pairwise traits. The upper triangle is the significant SNPs numbers in pairwise trits; The lower triangle is the potential candidate gene numbers in pairwise traits. The color bar represents the number of SNPs or candidate genes in pairwise traits.

To further confirm the relationships among agronomic traits at the whole genome level, 2526 significant SNPs $\left(p<1.707 \times 10^{-7}\right)$ were mapped independently on chromosomes $1-12$. We found that some of the SNPs clustered in a small region on a chromosome, which was deemed to be an SNP polymerization region. Totally, 37 SNP polymerization regions (total size: $264.69 \mathrm{Mbp}$ ) were found in the study, which could be selected areas in pepper breeding (Table S16). As shown in Figure 3, most SNP polymerization regions were distributed on chromosomes $1,6,9$, and 10 . The polymerization regions associated with more than four traits were mainly distributed on chromosomes 6-12 (Table S16). Compared with the correlation analysis results, we found that the stronger the correlation between two traits, the greater the possibility of them being in more than one polymerization region, and the higher the number of SNPs and genes in the same polymerization region (Figures 3-5 and Table S17). For example, Bh and Nfpa were negatively correlated (-0.59), and there were 18 different shared regions for them. Similar features were also found between Pt and Nfpa (16 regions), and Pt and Bh (15 regions) (Table S17).

\subsection{Verification of GWAS Results Based on Fine Mapping of Male-Sterility (Rf) Gene}

To verify the GWAS signals, we used molecular mapping of the $R f$ gene for cytoplasmic male sterility. In the $\mathrm{F}_{2}$ population of 2016, 255 individuals were fertile, and 79 were sterile. A chi-squared test revealed that the ratio of fertile:sterile individuals in this population was 3:1. The $R f$ gene was initially mapped within $4.9 \mathrm{cM}$ between the SSR markers P06-319 and PW6-146 (Figure 6a), with a physical distance of $1.8 \mathrm{Mb}$. In the $\mathrm{F}_{2}$ population of 2017, the recombinant single strains were screened with P06-319 and PW6-146 as two side markers, and 116 individuals were recombinant between these markers. Genotypic assay of the recombinants was performed using 10 SSR markers between P06-319 and PW6-146. Finally, the $R f$ gene was mapped to a region between the markers P06-247 and PW6-126. This region lies within 214,868,888-215,727,145 bp of chromosome 6 in the 'Zunla-1' genome, with a physical distance of $858,257 \mathrm{bp}$. Comparing the regions of molecular mapping with the GWAS signals, we found that the region of molecular mapping $(214,868,888-215,727,145)$ was highly 
consistent with that of GWAS $(214,443,037-215,536,517)$ (Figure 6b), which indicated that the GWAS signals were reliable.

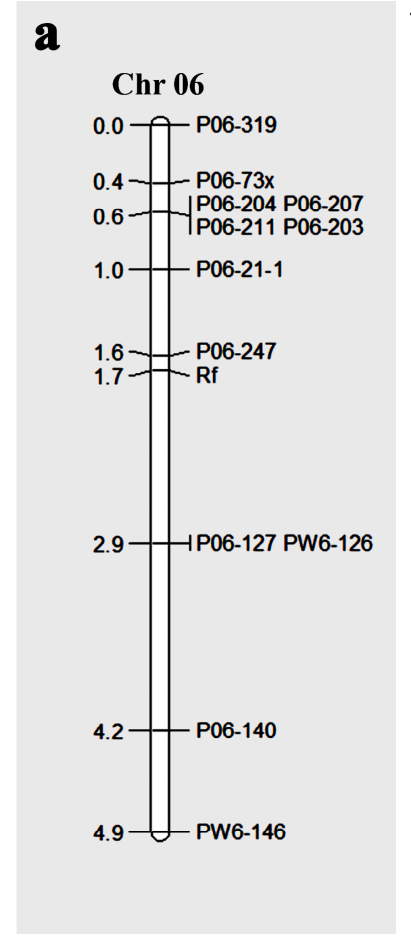

b

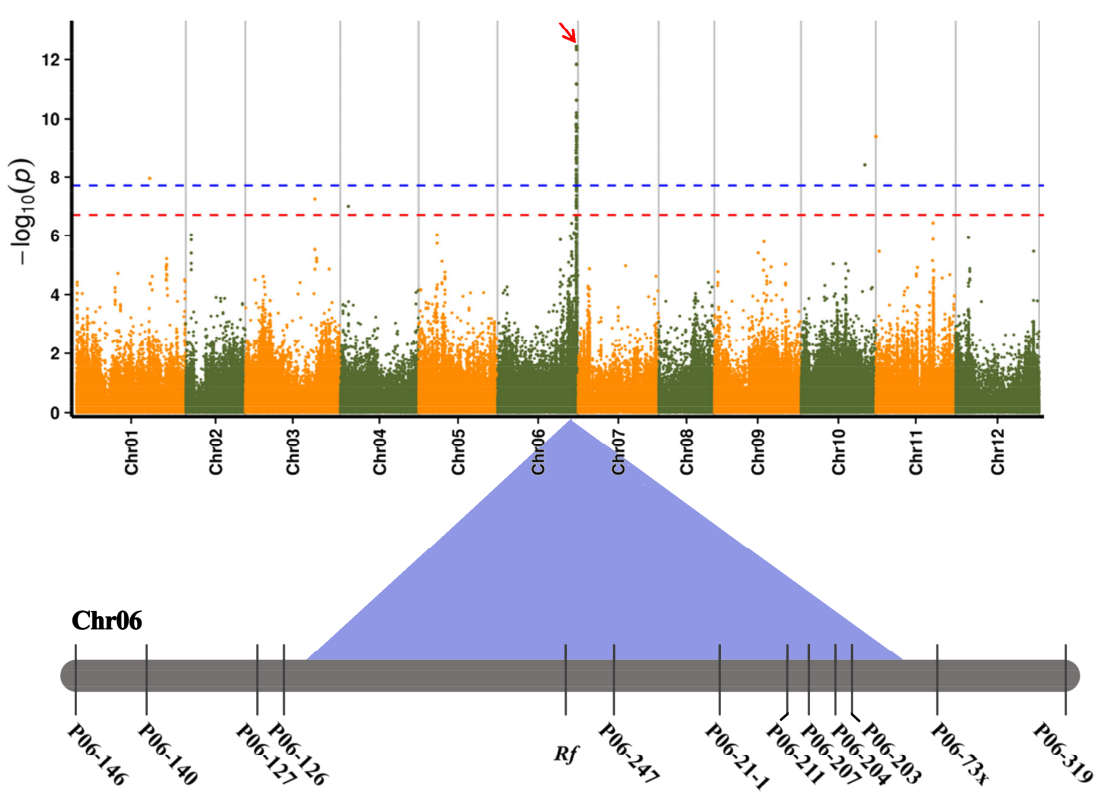

Figure 6. Comparison of molecular mapping with GWAS signals for Rf gene. (a) the molecular mapping result of Rf. (b) Comparison of the results of molecular mapping and GWAS mapping of Rf (factored spectrally transformed linear mixed (FaST-LMM) model). The red arrow represents the major peaks associated with male-sterility (Rf gene). The highly significant threshold is shown as a dash blue line $\left(p<1.707 \times 10^{-8}\right)$ and the significant threshold is shown as a dash red line $\left(p<1.707 \times 10^{-7}\right)$.

According to $R f$ gene localization, the 214,868,888-215,727,145-bp region of the 'Zunla-1' genome was annotated, and eight genes were found in this region (Table S13). cDNA of 138A and 138C were used as templates to detect whether there were differences in the coding sequences of the genes. The sequencing results showed that there were no differences in the coding sequences of the eight genes between the two templates.

The results of expression analysis showed that no expression was detected for Capana06g002963 and Capana06g002971. Capana06g002965 was expressed in both anthers and leaves, and its expression in anthers gradually decreased with bud development but showed no significant difference between 138A and 138C (Figure S21a). Capana06g002967 was mainly expressed in buds, and the expression level gradually increased with bud development. The expression level of $138 \mathrm{C}$ was significantly higher than that of 138A at all stages of bud development (Figure S21b). Capana06g002968 was mainly expressed in buds, and the highest expression was found in the anthers of 138A at the mature stage (Figure S21c). Capana06g002969 was expressed in both anthers and leaves, and the anther expression in all stages of 138C was significantly higher than that in 138A (Figure S21d). Capana06g002970 was expressed in both anthers and leaves, but there was no significant difference between 138A and 138C (Figure S21e). Capana06g002972 was expressed in both anthers and leaves, and the highest expression was in the anthers of 138C at maturity and was significantly higher than that of 138A (Figure S21f). Based on the results, Capana06g002967 and Capana06g002969 were identified as $R f$ candidate genes. 


\section{Discussion}

There are many agronomic traits with abundant phenotypes in pepper. However, most of the previous studies on the gene mapping of agronomic traits in pepper were based on the traditional QTL mapping method, and only a single or several traits were studied in the same study [48-51]. Although, there are also reports of the use of GWAS in pepper, most of this research was only focused on one or two traits [33,43,52]. In our study, the GWAS of 36 agronomic traits was carried out at the same time. A large number of SNP loci significantly associated with agronomic traits were obtained, and the main correlation peaks of some key agronomic traits were identified. Our results showed that GWAS could be used to study multiple agronomic traits in the same study, which greatly improved the efficiency of gene mapping of agronomic traits in pepper.

However, GWAS does have the potential for false positive errors due to the population structure, relatedness, and inappropriate statistical models [22,37,53,54]. From previous studies, we summarized three aspects that may contribute to improving the accuracy of GWAS results and that should be considered carefully, including population materials $[25,30,55,56]$, statistical methods [22,57], and validation methods $[43,52]$. Most previous studies only considered verification methods, not material population or statistical models. To minimize the rates of false positives and false negatives and to ensure the reliability of the GWAS results, we carried out four measures based on those three aspects. We used molecular mapping of the $R f$ gene for cytoplasmic male sterility as an example to verify GWAS signals. Comparing the regions of molecular mapping with GWAS signals, we found that the region of molecular mapping was highly consistent with that of GWAS, indicating that the GWAS signals were reliable. Furthermore, using SSR molecular marker technology combined with the GWAS results of 287 pepper samples, the $R f$ gene was finally located in the region of $858,257 \mathrm{bp}(214,868,888-215,727,145 \mathrm{bp})$ on chromosome 6 of the 'Zunla-1' genome, which was consistent with the two regions of 211.29-216.45 $\mathrm{Mb}$ and 215.11-215.68 $\mathrm{Mb}$ reported previously [58,59]. Additionally, in the simple comparison of the GWAS results of other traits with those of the traditional methods (Table S18), we found that more than one loci identified by the two methods (QTL and GWAS) for the same traits were located on the same chromosome, especially for some quantitative traits controlled by poly-genes, which also demonstrates the reliability of the GWAS result.

Pubescence is a key agronomic trait of pepper, which can improve drought resistance and protect plants from UV radiation, insects, and pathogenic bacteria [48,60]. Kim et al. (2010) found a major QTL (Ptl1) for stem pubescence on chromosome 10 [48]. Chunthawodtiporn et al. (2018) also found some QTL for pubescence on chromosomes 2, 10, and 11 and identified two candidate genes controlling pubescence formation on chromosome 10 [60]. In our study, pubescence was divided into Msp and Lp, and we detected correlation peaks for Msp on chromosomes 10 and 11, the latter being the major peak. However, the major peak associated with Lp was detected on chromosome 10. Those results showed that there was more than one major gene controlling the pubescence of pepper, and that genes may be specific to growth sites.

$\mathrm{Mfc}$ is an important agronomic trait of pepper. Our results showed that the major peak for Mfc was on chromosome 6, where the $y$ gene controls the red or yellow mature fruit [61]. However, the $c$-2 gene controlling red or orange mature fruit was detected on chromosome 4 [61]. The $c l$ gene, preventing chlorophyll degradation and controlling brown or olive-green mature fruit, was detected on chromosome 1 [62]. Aif is the stress response of pepper fruit to low temperature and strong UV radiation (Figure S4a), and its formation is mainly related to the accumulation of anthocyanin in fruit epidermis [63]. Genes regulating anthocyanin biosynthesis have been studied in many solanaceous plants, such as tomato, potato, eggplant, and pepper [64,65]. In pepper, a QTL for anthocyanin on immature fruit has been detected on chromosome 10, and a candidate gene was proposed for anthocyanin accumulation $[60,66]$. In this study, we found peaks of Aif on the same chromosome. Those results showed that the genetic and regulatory mechanism of fruit color is more complex, and there are differences among different mature fruit colors in pepper. 
Most agronomic traits of pepper are controlled by QTLs with complex correlations among them, causing difficulties for breeding programs and hindering the development of multi-trait pyramid breeding. Previous studies found that the genes controlling the color of different parts of pepper plants were aggregated on chromosomes and linked to the genes controlling fruit shape $[13,19,67]$. Ben Chaim et al. (2001) also found that markers in two genomic regions linked to QTL for Cucumber mosaic virus resistance were also linked to QTL for fruit weight [13]. Our study analyzed the relationship among 36 agronomic traits of pepper from different angles. First, the correlation analysis based on phenotypic data showed that agronomic traits were highly correlated with each other, and the most significant correlations were observed for fruit-related traits, which is consistent with the results from previous research [68]. Correlation studies can help to determine the traits on which selection should be based to breed peppers for specific purposes [69]. Second, we compared the distribution of peaks in the Manhattan plots and found that there was more than one shared peak among different traits, suggesting that the traits were linked, and controlled either by one pleiotropic gene or by a group of closely-linked genes. Hence, we further analyzed the distribution of SNPs that were significantly associated with the 36 agronomic traits and were found in SNP polymerization regions on chromosomes, consistent with previous research results [17]. Furthermore, 37 SNP polymerization regions were found, which may be the main regions of natural selection and artificial selection in the long-term evolution of pepper. These SNP polymerization regions can be the focus of molecular marker development in the process of multi-trait pyramid breeding to improve the efficiency of multi-trait pyramid breeding.

In summary, we obtained many SNPs associated with agronomic traits and genes near the SNPs, which provided a large amount of biological information for the study of agronomic traits in pepper. Furthermore, we comprehensively explored the correlations among different agronomic traits and found 37 SNP polymerization regions, which laid a theoretical foundation for future multi-trait pyramid breeding of pepper. In future research, we will analyze the genetic effect of SNPs in these regions and further clarify the association among different traits and the pleiotropism of candidate genes.

\section{Materials and Methods}

\subsection{Global Exploration of Correlations among the 36 Agronomic Traits}

In our study, we used 287 pepper accessions provided by the pepper research laboratory of the College of Horticulture, China Agricultural University for the global exploration of correlations among the 36 agronomic traits and the subsequent GWAS analysis. The 287 accessions were classified into C. baccatum (1), C. chinense (4), C. frutescens (4), and C. annuum (278). There were $13 \mathrm{~F}_{1}$ hybrids and 274 high-generation inbred lines (Table S2). All were planted three times with three replications in the greenhouses: once in 2017, 2018, and 2019. In each year, three replications (each replication with 12 plants) for each accession were grown in a randomized design in greenhouses and 3-6 plants were selected from each replication for surveying agronomic traits. The 36 agronomic traits were surveyed during different growing periods, details of the survey methods for agronomic traits are provided in Note S1 and Figures S1-S4.

Analysis of correlations among 36 agronomic traits was performed by the R software (version 3.5.0, Ross Ihaka and Robert Gentleman, University of Auckland, Auckland, New Zealand) base on the phenotypic data of three years. As shown in Table S2, highly consistent qualitative trait indicator data and mean values of nine replications over three year quantitative trait indicator were used for correlation analysis, because there was no extremely significant difference $(p<0.01)$ in their phenotypic data over 3 years (Table S2). Furthermore, the correlation network procedure was carried out using the R package "qgraph" [70]. 


\subsection{Analysis of Phenotypic Data}

Phenotypic variation of the population was analyzed and evaluated using the frequency distribution and $\mathrm{CV}$ of the phenotypes of each trait. To evaluate the population diversity of the 287 pepper accessions, the Shannon-Weaver diversity index $\left(\mathrm{H}^{\prime}\right)$ was calculated in Microsoft Excel 2007 version (MSO: 12.0.4518.1014) as follows:

$$
\mathrm{H}^{\prime}=-\sum_{i=1}^{S} P i \ln P i
$$

where $P_{i}$ is the percentage of materials contained in class $i$ for a quantitative trait type indicator. For qualitative trait type indices, $P i$ is the percentage of materials, of which, phenotype was assigned a value equal to $i$ (details of assignment standards are shown in Table S19). $\mathrm{S}$ is the number of grades for quantitative indicators, or the number of assigned values for qualitative trait type indicators.

Descriptive statistics (e.g., mode, mean, and max) were calculated and graphs drawn in Microsoft Excel 2007. Results are presented as the mean of nine replications over 3 years.

\subsection{Extraction of Genomic DNA}

Genomic DNA was isolated from six fresh leaves from plants using the cetyltrimethylammonium bromide method [71,72]. A NanoDrop 2000 spectrophotometer (Thermo Scientific, Waltham, MA, USA) was used to determine the DNA concentration and quality to ensure that DNA samples met the requirements of the sequencing reaction (concentration $\geq 20 \mathrm{ng} / \mu \mathrm{L}$; volume $\geq 30 \mu \mathrm{L}$ ).

\subsection{Design of Enzyme Digestion Scheme}

The genome of 'Zunla-1' (http://peppersequence.genomics.cn/page/species/index.jsp, version 2.0) was selected as the reference genome for an electronic digestion prediction experiment. The genome size of 'Zunla-1' was about $3.36 \mathrm{~Gb}$, and GC content was $34.97 \%$. The principles for selection of the enzyme digestion scheme were as follows: (1) the proportion of enzyme-digested fragments located in repeated sequences was as low as possible; (2) distribution of enzyme-digested fragments throughout the genome was as even as possible; (3) length of enzyme-digested fragments was in good agreement with the experimental system; (4) number of enzyme-digested fragments (SLAF tags) reached the expected number $(328,885)$ of SLAF tags.

\subsection{Construction of SLAF Libraries and High-Throughput Sequencing}

According to the results of the electron prediction experiment, HaeIII (New England Biolabs, Ipswich, MA, USA) was used to separately digest the eligible genomic DNA. Single-nucleotide A overhangs from these DNA fragments were polished using the Klenow fragment (New England Biolabs), and fragments were then ligated to dual-index sequencing adaptors. Adaptor-ligated fragments were then amplified by PCR, purified, pooled, and screened to construct the SLAF library [43,73]. SLAF library construction and screening were performed as described by Sun et al. (2013) [74]. From the quality-tested library, target DNA fragments of sizes from 314 to $464 \mathrm{bp}$ (SLAF) were selected for paired-end sequencing on an Illumina HiSeq 2500 platform (Illumina Inc., San Diego, CA, USA) at Beijing Biomarker Technologies Corporation in Beijing, China. Additionally, we set the control genome (Oryza sativa spp. japonica; $374.30 \mathrm{M}$; http://rapdb.dna.affrc.go.jp/) to the same sequencing process, to verify whether the experimental process was reliable. The paired-end comparison efficiency of control data was $95.65 \%$, and the enzyme digestion efficiency was $94.74 \%$, showing that the construction of SLAF libraries was normal. 


\subsection{Identification and Distribution Analysis of Labels (SLAF Tags and SNPS)}

Raw sequencing reads of SLAF were filtered for quality and trimmed to remove adaptors. The $Q$ value was used to evaluate the single-base error rate of high-throughput sequencing. A $Q$ value of $30(\mathrm{Q} 30, \mathrm{Q}<30)$ represents a $0.1 \%$ error rate or $99.9 \%$ sequence accuracy. Then, the proportion of sequencing quality scores at $\mathrm{Q}_{30}$ in the SLAF libraries and GC content were used to evaluate the quality of sequencing. The formula for calculating the Q30 value is listed below:

$$
\mathrm{Q}_{30}=-10 \times \log _{10} \mathrm{P}
$$

where $P$ is the base sequencing error rate.

Furthermore, we clustered all paired-end reads that had perfect index reads according to sequence similarity with the genome of 'Zunla- 1 '. Reads with $>90 \%$ identity were grouped into a single SLAF tag, and SLAF tags with a sequence that varied from sample to sample were defined as polymorphic SLAF tags [43].

Sequencing reads were aligned to the pepper reference genome ('Zunla-1') using BWA software; local realignments were conducted, and SNPs were detected using GATK software [75]. To ensure the accuracy of the SNPs identified using GATK, SAMtools software also was used to detect SNPs [76]. The intersection of SNPs that were detected using the two methods was designated as the final SNPs and used for further analysis. Distribution of SLAF tags and SNPs was analyzed by R (version 3.5.0, Ross Ihaka and Robert Gentleman) and SPSS software (version 22.0, International Business Machines Corporation, Armonk, New York, USA). Graphs were drawn in R (version 3.5.0, Ross Ihaka and Robert Gentleman).

\subsection{Population Structure Analysis}

Based on the filtered SNPs (integrity > 0.5; MAF > 0.05), phylogeny analysis, PCA, and population analysis were performed in turn. All of the filtered SNPs $(594,429)$ were genotyped by MEGA6 software and used for the construction of the phylogenetic tree by the neighbor-joining algorithm [77]. PCA was performed using the smartPCA program from the EIGENSOFT package (https://github. com/DReichLab/EIG; v.6.0.1) [78]. Population structure of 287 accessions was calculated using admixture software [79]. The analysis used 594,429 SNPs of 287 accessions to infer the genetic background of an accession that belongs to a cluster under a given number of subgroups (K). The number of genetic clusters was predefined as $K=1-15$ for all accessions to explore the population structure. The relatedness coefficient was counted using SPAGeDi (version 1.4C) software [80]. Linkage disequilibrium between SNPs was estimated with $\mathrm{R}^{2}$ using HAPLOVIEW software (https: //www.broadinstitute.org/ftp/pub/mpg/haploview/hapinstall.exe) [81].

\subsection{Genome-Wide Association Analyses of 36 Agronomic Traits}

Total filtered SNPs (integrity > 0.5; MAF > 0.05) detected from 287 accessions were used for GWAS. GWAS for all traits (based on GLM, MIM, CMLM, FaST-LMM, and EMMAX models) was conducted using TASSEL (http://www.maizegenetics.net/tassel), FaST-LMM (https://www.microsoft. com/en-us/download/confirmation.aspx?id=52588), and EMMAX (http://csg.sph.umich.edu//kang/ emmax/download/index.html) software with default settings [82-84]. Details of those model formulae are as follows:

The general linear model formula was:

$$
\mathrm{y}=\mathrm{X} \alpha+\mathrm{Q} \beta+\mathrm{e}
$$

The mixed linear model formula was:

$$
\mathrm{y}=\mathrm{X} \alpha+\mathrm{Q} \beta+\mathrm{K} \mu+\mathrm{e}
$$


where $y$ is the phenotype (for qualitative traits, phenotypic data were the same for 2017, 2018 and 2019; for quantitative traits, it was the mean of nine replications over three years, as shown in Table S2), $\mathrm{X}$ is the genotype, $\mathrm{Q}$ is the $\mathrm{Q}$ matrix, and $\mathrm{K}$ is the relative relatedness matrix. $\mathrm{X} \alpha$ and $\mathrm{Q} \beta$ are fixed effects, and $K \mu$ and e are random effects. The $Q$ value, which represents population structure, was calculated by the admixture software [79]. The $\mathrm{K}$ value, which represents the relative relatedness between accessions, was calculated by the SPAGeDi software (version 1.4C) [80].

The $p$ value was calculated for each SNP, and $p<1.707 \times 10^{-8}(p=0.01 / n ; n=$ total markers used, which is roughly a Bonferroni correction, corresponding to $-\log 10(p)=8$, blue line) and $p<1.707 \times 10^{-7}$ $(p=0.1 / n ; n=$ total markers used, which is roughly a Bonferroni correction, corresponding to $-\log 10$ $(p)=7$, red line) was defined as the suggestive (highly significant) threshold and genome-wide control (significant) threshold, respectively. The genes within $100 \mathrm{~kb}$ up- or down-stream of these significant SNPs were found by Jbrowser in SGN (Sol Genomics Network https://solgenomics.net/) and reported as potential candidate genes [46,47]. The Manhattan and QQ plots of GWAS were drawn using the $R$ package "qqman.". The heatmaps of the SNPs and genes number in pairwise traits and the physical map of significant SNPs (or polymerization regions) associated with 26 traits were drawn by R software (version 3.5.0, Ross Ihaka and Robert Gentleman).

\subsection{Molecular Mapping of the Nuclear Fertility-Restoring Gene (Rf) for Cytoplasmic Male Sterility}

Two $\mathrm{F}_{2}$ populations were constructed separately in spring 2016 (334 individuals) and spring 2017 (5026 individuals) for fine mapping, with parents (17C641 and 17C643) selected from the GWAS population. In these two populations, the 2016 population was used for initial mapping, and the 2017 population was subsequently used for fine mapping. All individuals were grown inside greenhouses with an inter-individual distance of $30 \mathrm{~cm}$, and unified field management was used. Methodological details are provided in Note S1.

A genotypic assay was performed using SSR markers (Table S20). The SSR markers that cover the entire pepper genome were designed according to the published pepper genome sequence. The SSR loci that covered the entire genome were developed using a whole $C$. annuum 'Zunla- 1 ' genome scan of double-base repeats, and SSR loci that were repeated six times or more developed using misa.pl.300bp software were extended using Python scripts on each side of the SSR loci. The primers were designed using Primer 3 (Premier Biosoft International, Palo Alto, CA, USA). The PCR solution was $10 \mu \mathrm{L}$ in volume and contained $1.0 \mu \mathrm{L}$ DNA template, $0.5 \mu \mathrm{L}$ of both forward and reverse primers, $5.0 \mu \mathrm{L}$ Taq

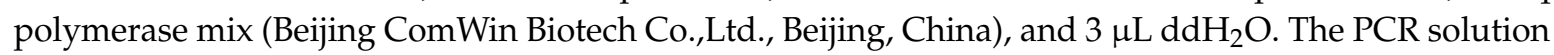
was $10 \mu \mathrm{L}$ and contained $1.0 \mu \mathrm{L}$ DNA template, $0.5 \mu \mathrm{L}$ of both forward and reverse primers, $5.0 \mu \mathrm{L}$ Taq polymerase mix, and $3 \mu \mathrm{LddH} \mathrm{d}_{2} \mathrm{O}$. The PCR protocol was as follows: initial denaturation at $94^{\circ} \mathrm{C}$ for $5 \mathrm{~min} ; 35$ cycles of denaturation at $94{ }^{\circ} \mathrm{C}$ for $30 \mathrm{~s}$, annealing at $53{ }^{\circ} \mathrm{C}$ for $45 \mathrm{~s}$, and extension at $72{ }^{\circ} \mathrm{C}$ for $30 \mathrm{~s}$, before a final extension at $72{ }^{\circ} \mathrm{C}$ for $5 \mathrm{~min}$. PCR products were stored at $4{ }^{\circ} \mathrm{C}$ and analyzed using $7 \%$ polyacrylamide gel electrophoresis. Joinmap 4.0 software (Beijing Lucidus Bioinformation Technology Co., Ltd., Beijing, China) was used to analyze the linkage relationship between the markers and the $R f$ gene. RT-PCR, real-time PCR, and sequencing of PCR products were performed as described by Wang et al. (2019) [85].

\section{Conclusions}

Based on the SLAF-seq, the GWAS of 36 agronomic traits in 287 pepper accessions was performed in this study. A total of 1,824,874 SLAF tags, 1,025,395 polymorphism SLAF tags and 9,557,790 SNPs, were obtained. Those tags/labels were normally distributed in the population consisted of 287 peppers. Meanwhile, those tags/labels were uniformed distributed on the 12 pepper chromosomes, indicating those SLAF tags and SNP developed can be used for GWAS. In order to obtain a better result, Fast-LMM, EMMAX and GLM were selected as the optimal statistical models for certain traits. In our study, a total of 1487 SNP, 2126 candidate genes and 109 correlation peaks were significantly $\left(p<1.707 \times 10^{-8}\right)$ correlated with the 26 agronomic traits in pepper by GWAS. Thirty-seven SNP polymerization regions 
were obtained from the correlation analysis. The two traits with higher correlation index may locate in a same SNP polymerization region. These results is useful for multi-trait pyramid breeding of pepper. In addition, based on the gene annotation and expression patterns, Capana06g002967 and Capana06g002969 were identified as the candidates for the $R f$ gene, which provided a reference for the further study of CMS in Capsicum.

Supplementary Materials: Supplementary Materials can be found at http://www.mdpi.com/1422-0067/20/22/ 5675/s1.

Author Contributions: L.W. investigated agronomic traits, analyzed the data, and drafted the manuscript. P.W. performed the fine mapping of the $R f$ gene and the qRT-PCR analysis. L.S. drew the figures and modified the manuscript. Q.C. participated in surveying agronomic traits. Q.L., T.L., and J.L. participated in DNA extraction. Y.A. and Y.W. participated in RNA extraction. W.Y. revised the manuscript. H.S. designed and directed the entire project. All authors have read and approved the final manuscript.

Funding: This work was carried out with the support of The National Key Research and Development Program of China (2016YFD0101704), The Beijing Fruit Vegetables Innovation Team of Modern Agricultural Industry Technology System (BAIC01-2019) and The Construction of Beijing Science and Technology Innovation and Service Capacity in Top Subjects (CEFF-PXM2019-014207-000032).

Acknowledgments: We would like to thank Editage [www.editage.cn] for English language editing.

Conflicts of Interest: The authors declare that the research was conducted in the absence of any commercial or financial relationships that could be construed as a potential conflict of interest.

\section{References}

1. International Plant Genetic Resources, I. Descriptors for Capsicum (Capsicum spp.); Bioversity International: Rome, Italy, 1995.

2. Junior, E.; Silva, W.C.; de Carvalho, S.I.C.; Duarte, J.B. Identification of minimum descriptors for characterization of Capsicum spp. germplasm. Hortic. Bras. 2013, 31, 190-202.

3. Li, X.; Zhang, B. (Eds.) Descriptors and Data Standard for Capisicum (Capisicum annum L.; Capisicum frutescens L.; Capisicum chinense, Capisicum bacctum, Capisicum pubescens), 1st ed.; China Agriculture Press, Ltd.: Beijing, China, 2006. (In Chinese)

4. Danojevic, D.; Medic-Pap, S. Different multivariate analysis for fruit traits in sweet pepper breeding. Genet. Belgrade 2018, 50, 121-129. [CrossRef]

5. Rao, V.V.R.; Jaisani, B.G.; Patel, G.J. Interrelationship and path coefficients of quantitative traits in Chilli. Indian J. Agric. Sci. 1974, 44, 462-465.

6. Gill, H.S.; Asawa, B.M.; Thakur, P.C.; Thakur, T.C. Correlation, path-coefficient and multiple-regression analysis in sweetpepper. Indian J. Agric. Sci. 1977, 47, 408-410.

7. Gupta, C.R.; Yadav, R.D.S. Genetic variability and path analysis in chili capsicum-annuum. Genet. Agrar. $1984,38,425-432$.

8. Espigares, T.; Peco, B. Mediterranean annual pasture dynamics-Impact of autumn drought. J. Ecol. 1995, 83, 135-142. [CrossRef]

9. Hagley, E.A.C.; Bronskill, J.F.; Ford, E.J. Effect of the physical nature of leaf and fruit surfaces on oviposition by the codling moth, cydia-pomonella (lepidoptera, tortricidae). Can. Entomol. 1980, 112, 503-510. [CrossRef]

10. Valverde, P.L.; Fornoni, J.; Nunez-Farfan, J. Defensive role of leaf trichomes in resistance to herbivorous insects in Datura stramonium. J. Evol. Biol. 2001, 14, 424-432. [CrossRef]

11. Tewksbury, J.J.; Levey, D.J.; Huizinga, M.; Haak, D.C.; Traveset, A. Costs and benefits of capsaicin-mediated control of gut retention in dispersers of wild chilies. Ecology 2008, 89, 107-117. [CrossRef]

12. Tewksbury, J.J.; Nabhan, G.P. Seed dispersal—Directed deterrence by capsaicin in chillies. Nature 2001, 412, 403-404. [CrossRef]

13. Ben Chaim, A.; Paran, I.; Grube, R.C.; Jahn, M.; van Wijk, R.; Peleman, J. QTL mapping of fruit-related traits in pepper (Capsicum annuum). Theor. Appl. Genet. 2001, 102, 1016-1028. [CrossRef]

14. Blum, E.; Mazourek, M.; O'Connell, M.; Curry, J.; Thorup, T.; Liu, K.D.; Jahn, M.; Paran, I. Molecular mapping of capsaicinoid biosynthesis genes and quantitative trait loci analysis for capsaicinoid content in Capsicum. Theor. Appl. Genet. 2003, 108, 79-86. [CrossRef] [PubMed] 
15. Rao, G.U.; Chaim, A.B.; Borovsky, Y.; Paran, I. Mapping of yield-related QTLs in pepper in an interspecific cross of Capsicum annuum and C-frutescens. Theor. Appl. Genet. 2003, 106, 1457-1466. [CrossRef] [PubMed]

16. Yarnes, S.C.; Ashrafi, H.; Reyes-Chin-Wo, S.; Hill, T.A.; Stoffel, K.M.; van Deynze, A. Identification of QTLs for capsaicinoids, fruit quality, and plant architecture-related traits in an interspecific Capsicum RIL population. Genome 2013, 56, 61-74. [CrossRef]

17. Han, K.; Jeong, H.-J.; Yang, H.-B.; Kang, S.-M.; Kwon, J.-K.; Kim, S.; Choi, D.; Kang, B.-C. An ultra-high-density bin map facilitates high-throughput QTL mapping of horticultural traits in pepper (Capsicum annuum). DNA Res. 2016, 23, 81-91. [CrossRef]

18. Li, X.; Wu, L.; Wang, J.; Sun, J.; Xia, X.; Geng, X.; Wang, X.; Xu, Z.; Xu, Q. Genome sequencing of rice subspecies and genetic analysis of recombinant lines reveals regional yield- and quality-associated loci. BMC Biol. 2018, 16, 102. [CrossRef]

19. Peterson, P.A. Linkage of fruit shape and color genes in capsicum. Genetics 1959, 44, 407-419.

20. Chaim, A.B.; Borovsky, Y.; de Jong, W.; Paran, I. Linkage of the A locus for the presence of anthocyanin and fs10.1, a major fruit-shape QTL in pepper. Theor. Appl. Genet. 2003, 106, 889-894. [CrossRef]

21. Salvi, S.; Tuberosa, R. To clone or not to clone plant QTLs: Present and future challenges. Trends Plant Sci. 2005, 10, 297-304. [CrossRef]

22. Yu, J.M.; Buckler, E.S. Genetic association mapping and genome organization of maize. Curr. Opin. Biotechnol. 2006, 17, 155-160. [CrossRef]

23. Ngan Thi, P.; Sim, S. Genomic tools and their implications for vegetable breeding. Korean J. Hortic. Sci. Technol. 2017, 35, 149-164.

24. Ingvarsson, P.K.; Street, N.R. Association genetics of complex traits in plants. New Phytol. 2011, 189, 909-922. [CrossRef] [PubMed]

25. Huang, X.; Wei, X.; Sang, T.; Zhao, Q.; Feng, Q.; Zhao, Y.; Li, C.; Zhu, C.; Lu, T.; Zhang, Z.; et al. Genome-wide association studies of 14 agronomic traits in rice landraces. Nat. Genet. 2010, 42, 961. [CrossRef] [PubMed]

26. Upadhyaya, H.D.; Bajaj, D.; Narnoliya, L.; Das, S.; Kumar, V.; Gowda, C.L.L.; Sharma, S.; Tyagi, A.K.; Parida, S.K. Genome-Wide Scans for Delineation of Candiate Genes Regulating Seed-Protein Content in Chickpea. Front. Plant Sci. 2016, 7, 302. [CrossRef] [PubMed]

27. Navarro, J.A.R.; Willcox, M.; Burgueno, J.; Romay, C.; Swarts, K.; Trachsel, S.; Preciado, E.; Terron, A.; Delgado, H.V.; Vidal, V.; et al. A study of allelic diversity underlying flowering-time adaptation in maize landraces. Nat. Genet. 2017, 49, 476-480. [CrossRef] [PubMed]

28. Sonah, H.; O'Donoughue, L.; Cober, E.; Rajcan, I.; Belzile, F. Identification of loci governing eight agronomic traits using a GBS-GWAS approach and validation by QTL mapping in soya bean. Plant Biotechnol. J. 2015, 13, 211-221. [CrossRef]

29. Sauvage, C.; Segura, V.; Bauchet, G.; Stevens, R.; Thi, D.P.; Nikoloski, Z.; Fernie, A.R.; Causse, M. Genome-Wide Association in Tomato Reveals 44 Candidate Loci for Fruit Metabolic Traits. Plant Physiol. 2014, 165, 1120-1132. [CrossRef]

30. Zhao, J.; Artemyeva, A.; del Carpio, D.P.; Basnet, R.K.; Zhang, N.; Gao, J.; Li, F.; Bucher, J.; Wang, X.; Visser, R.G.F.; et al. Design of a Brassica rapa core collection for association mapping studies. Genome 2010, 53, 884-898. [CrossRef]

31. Zhang, P.; Zhu, Y.; Wang, L.; Chen, L.; Zhou, S. Mining candidate genes associated with powdery mildew resistance in cucumber via super-BSA by specific length amplified fragment (SLAF) sequencing. BMC Genom. 2015, 16, 1058. [CrossRef]

32. Sakiroglu, M.; Brummer, E.C. Identification of loci controlling forage yield and nutritive value in diploid alfalfa using GBS-GWAS. Theor. Appl. Genet. 2017, 130, 261-268. [CrossRef]

33. Nimmakayala, P.; Abburi, V.L.; Abburi, L.; Alaparthi, S.B.; Cantrell, R.; Park, M.; Choi, D.; Hankins, G.; Malkaram, S.; Reddy, U.K. Linkage disequilibrium and population-structure analysis among Capsicum annuum L. cultivars for use in association mapping. Mol. Genet. Genom. 2014, 289, 513-521. [CrossRef] [PubMed]

34. Nimmakayala, P.; Abburi, V.L.; Saminathan, T.; Alaparthi, S.B.; Almeida, A.; Davenport, B.; Nadimi, M.; Davidson, J.; Tonapi, K.; Yadav, L.; et al. Genome-wide Diversity and Association Mapping for Capsaicinoids and Fruit Weight in Capsicum annuum L. Sci. Rep. 2016, 6, 38081. [CrossRef] [PubMed]

35. Nimmakayala, P.; Abburi, V.L.; Saminathan, T.; Almeida, A.; Davenport, B.; Davidson, J.; Reddy, C.V.C.M.; Hankins, G.; Ebert, A.; Choi, D.; et al. Genome-Wide Divergence and Linkage Disequilibrium Analyses for 
Capsicum baccatum Revealed by Genome-Anchored Single Nucleotide Polymorphisms. Front. Plant Sci. Sci. 2016, 7, 1646. [CrossRef] [PubMed]

36. Ahn, Y.-K.; Manivannan, A.; Karna, S.; Jun, T.-H.; Yang, E.-Y.; Choi, S.; Kim, J.-H.; Kim, D.-S.; Lee, E.-S. Whole Genome Resequencing of Capsicum baccatum and Capsicum annuum to Discover Single Nucleotide Polymorphism Related to Powdery Mildew Resistance. Sci. Rep. 2018, 8, 5188. [CrossRef] [PubMed]

37. Zhu, C.; Gore, M.; Buckler, E.S.; Yu, J. Status and Prospects of Association Mapping in Plants. Plant Genome 2008, 1, 5-20. [CrossRef]

38. Brachi, B.; Faure, N.; Horton, M.; Flahauw, E.; Vazquez, A.; Nordborg, M.; Bergelson, J.; Cuguen, J.; Roux, F. Linkage and Association Mapping of Arabidopsis thaliana Flowering Time in Nature. PLoS Genet. 2010, 6, e1000940. [CrossRef] [PubMed]

39. Crowell, S.; Korniliev, P.; Falcao, A.; Ismail, A.; Gregorio, G.; Mezey, J.; McCouch, S. Genome-wide association and high-resolution phenotyping link Oryza sativa panicle traits to numerous trait-specific QTL clusters. Nat. Commun. 2016, 7, 10527. [CrossRef]

40. Sallam, A.; Arbaoui, M.; El-Esawi, M.; Abshire, N.; Martsch, R. Identification and Verification of QTL Associated with Frost Tolerance Using Linkage Mapping and GWAS in Winter Faba Bean. Front. Plant Sci. 2016, 7, 1098. [CrossRef]

41. Tian, F.; Bradbury, P.J.; Brown, P.J.; Hung, H.; Sun, Q.; Flint-Garcia, S.; Rocheford, T.R.; McMullen, M.D.; Holland, J.B.; Buckler, E.S. Genome-wide association study of leaf architecture in the maize nested association mapping population. Nat. Genet. 2011, 43, 159. [CrossRef]

42. Han, K.; Lee, H.-Y.; Ro, N.-Y.; Hur, O.-S.; Lee, J.-H.; Kwon, J.-K.; Kang, B.-C. QTL mapping and GWAS reveal candidate genes controlling capsaicinoid content in Capsicum. Plant Biotechnol. J. 2018, 16, 1546-1558. [CrossRef]

43. Zhang, X.; Wang, G.; Chen, B.; Du, H.; Zhang, F.; Zhang, H.; Wang, Q.; Geng, S. Candidate genes for first flower node identified in pepper using combined SLAF-seq and BSA. PLoS ONE 2018, 13, e0194071. [CrossRef] [PubMed]

44. Xu, X.; Chao, J.; Cheng, X.; Wang, R.; Sun, B.; Wang, H.; Luo, S.; Xu, X.; Wu, T.; Li, Y. Mapping of a Novel Race Specific Resistance Gene to Phytophthora Root Rot of Pepper (Capsicum annuum) Using Bulked Segregant Analysis Combined with Specific Length Amplified Fragment Sequencing Strategy. PLoS ONE 2016, 11, e0151401. [CrossRef] [PubMed]

45. Guo, G.; Wang, S.; Liu, J.; Pan, B.; Diao, W.; Ge, W.; Gao, C.; Snyder, J.C. Rapid identification of QTLs underlying resistance to Cucumber mosaic virus in pepper (Capsicum frutescens). Theor. Appl. Genet. 2017, 130, 41-52. [CrossRef] [PubMed]

46. Wu, D.; Liang, Z.; Yan, T.; Xu, Y.; Xuan, L.; Tang, J.; Zhou, G.; Lohwasser, U.; Hua, S.; Wang, H.; et al. Whole-genome resequencing of a worldwide collection of Rapeseed accessions reveals the genetic basis of ecotype divergence. Mol. Plant 2019, 12, 30-43. [CrossRef] [PubMed]

47. Lu, K.; Wei, L.; Li, X.; Wang, Y.; Wu, J.; Liu, M.; Zhang, C.; Chen, Z.; Xiao, Z.; Jian, H.; et al. Whole-genome resequencing reveals Brassica napus origin and genetic loci involved in its improvement. Nat. Commun. 2019, 10, 1154. [CrossRef] [PubMed]

48. Kim, H.J.; Han, J.-H.; Kwon, J.-K.; Park, M.; Kim, B.-D.; Choi, D. Fine mapping of pepper trichome locus 1 controlling trichome formation in Capsicum annuum L. CM334. Theor. Appl. Genet. 2010, 120, 1099-1106. [CrossRef] [PubMed]

49. Lee, H.-R.; Kim, K.-T.; Kim, H.-J.; Han, J.-H.; Kim, J.-H.; Yeom, S.-I.; Kim, H.J.; Kang, W.-H.; Jinxia, S.; Park, S.-W.; et al. QTL analysis of fruit length using rRAMP, WRKY, and AFLP markers in chili pepper. Hortic. Environ. Biotechnol. 2011, 52, 602-613. [CrossRef]

50. Lee, J.; Park, S.J.; Hong, S.C.; Han, J.-H.; Choi, D.; Yoon, J.B. QTL mapping for capsaicin and dihydrocapsaicin content in a population of Capsicum annuum "NB1'xCapsicum chinense" Bhut Jolokia'. Plant Breed. 2016, 135, 376-383. [CrossRef]

51. Dwivedi, N.; Kumar, R.; Paliwal, R.; Kumar, U.; Kumar, S.; Singh, M.; Singh, R.K. QTL mapping for important horticultural traits in pepper (Capsicum annuum L.). J. Plant Biochem. Biotechnol. 2015, 24, 154-160. [CrossRef]

52. Siddique, M.I.; Lee, H.-Y.; Ro, N.-Y.; Han, K.; Venkatesh, J.; Solomon, A.M.; Patil, A.S.; Changkwian, A.; Kwon, J.-K.; Kang, B.-C. Identifying candidate genes for Phytophthora capsici resistance in pepper (Capsicum annuum) via genotyping-by-sequencing-based QTL mapping and genome-wide association study. Sci. Rep. 2019, 9, 1-15. [CrossRef] 
53. Korte, A.; Farlow, A. The advantages and limitations of trait analysis with GWAS: A review. Plant Methods 2013, 9, 29. [CrossRef] [PubMed]

54. Xiao, Y.; Liu, H.; Wu, L.; Warburton, M.; Yan, J. Genome-wide Association Studies in Maize: Praise and Stargaze. Mol. Plant 2017, 10, 359-374. [CrossRef] [PubMed]

55. Li, X.; Yan, W.; Agrama, H.; Jia, L.; Shen, X.; Jackson, A.; Moldenhauer, K.; Yeater, K.; McClung, A.; Wu, D. Mapping QTLs for improving grain yield using the USDA rice mini-core collection. Planta 2011, 234, 347-361. [CrossRef] [PubMed]

56. Jiang, H.; Huang, L.; Ren, X.; Chen, Y.; Zhou, X.; Xia, Y.; Huang, J.; Lei, Y.; Yan, L.; Wan, L.; et al. Diversity characterization and association analysis of agronomic traits in a Chinese peanut (Arachis hypogaea L.) mini-core collection. J. Integr. Plant Biol. 2014, 56, 159-169. [CrossRef]

57. Xie, D.; Dai, Z.; Yang, Z.; Tang, Q.; Sun, J.; Yang, X.; Song, X.; Lu, Y.; Zhao, D.; Zhang, L.; et al. Genomic variations and association study of agronomic traits in flax. BMC Genom. 2018, 19, 512. [CrossRef]

58. Wang, N. QTL Analysis and Effect of Different Cultivation Conditions on Restoration of Cytoplasmic Male Sterility in Capsicum. D; Chinese Academy of Agricultural Sciences: Beijing, China, 2015. (In Chinese)

59. Chen, J. Development and Application of Genome-Wide SSR and SNP Markers in Pepper (Capsicum spp.), D; SouthChina Agricultural University: Guangzhou, China, 2016. (In Chinese)

60. Chunthawodtiporn, J.; Hill, T.; Stoffel, K.; van Deynze, A. Quantitative Trait Loci Controlling Fruit Size and Other Horticultural Traits in Bell Pepper (Capsicum annuum). Plant Genome 2018, 11. [CrossRef]

61. Thorup, T.A.; Tanyolac, B.; Livingstone, K.D.; Popovsky, S.; Paran, I.; Jahn, M. Candidate gene analysis of organ pigmentation loci in the Solanaceae. Proc. Natl. Acad. Sci. USA 2000, 97, 11192-11197. [CrossRef]

62. Efrati, A.; Eyal, Y.; Paran, I. Molecular mapping of the chlorophyll retainer (cl) mutation in pepper (Capsicum spp.) and screening for candidate genes using tomato ESTs homologous to structural genes of the chlorophyll catabolism pathway. Genome 2005, 48, 347-351. [CrossRef]

63. Lightbourn, G.J.; Griesbach, R.J.; Novotny, J.A.; Clevidence, B.A.; Rao, D.D.; Stommel, J.R. Effects of anthocyanin and carotenoid combinations on foliage and immature fruit color of Capsicum annuum L. J. Hered. 2008, 99, 105-111. [CrossRef]

64. De Jong, W.S.; Eannetta, N.T.; de Jong, D.M.; Bodis, M. Candidate gene analysis of anthocyanin pigmentation loci in the Solanaceae. Theor. Appl. Genet. 2004, 108, 423-432. [CrossRef]

65. Rinaldi, R.; van Deynze, A.; Portis, E.; Rotino, G.L.; Toppino, L.; Hill, T.; Ashrafi, H.; Barchi, L.; Lanteri, S. New Insights on Eggplant/Tomato/Pepper Synteny and Identification of Eggplant and Pepper Orthologous QTL. Front. Plant Sci. 2016, 7, 1031. [CrossRef] [PubMed]

66. Borovsky, Y.; Paran, I. Characterization of fs10.1, a major QTL controlling fruit elongation in Capsicum. Theor. Appl. Genet. 2011, 123, 657-665. [CrossRef] [PubMed]

67. Ben Chaim, A.; Borovsky, Y.; Rao, G.U.; Tanyolac, B.; Paran, I. fs3.1, a major fruit shape QTL conserved in Capsicum. Genome 2003, 46, 1-9. [CrossRef] [PubMed]

68. da Silva, A.R.; Rego, E.R.d.; Pessoa, A.M.D.S.; Rego, M.M.D. Correlation network analysis between phenotypic and genotypic traits of chili pepper. Pesqui. Agropecu. Bras. 2016, 51, 372-377. [CrossRef]

69. Olawuyi, O.J.; Jonathan, S.G.; Babatunde, F.E.; Babalola, B.J.; Yaya, O.O.S.; Agbolade, J.O.; Aina, D.A.; Egun, C.J. Accession * treatment interaction, variability and correlation studies of pepper (Capsicum spp.) under the influence of arbuscular mycorrhiza fungus (Glomus clarum) and cow dung. Am. J. Plant Sci. 2014, 5, 683-690. [CrossRef]

70. Epskamp, S.; Cramer, A.O.J.; Waldorp, L.J.; Schmittmann, V.D.; Borsboom, D. qgraph: Network Visualizations of Relationships in Psychometric Data. J. Stat. Softw. 2012, 48, 1-18. [CrossRef]

71. Fulton, T.M.; Chunwongse, J.; Tanksley, S.D. Microprep protocol for extraction of DNA from tomato and other herbaceous plants. Plant Mol. Biol. Report. 1995, 13, 207-209. [CrossRef]

72. Lee, J.-H.; An, J.-T.; Siddique, M.I.; Han, K.; Choi, S.; Kwon, J.-K.; Kang, B.-C. Identification and molecular genetic mapping of Chili veinal mottle virus (ChiVMV) resistance genes in pepper (Capsicum annuum). Mol. Breed. 2017, 37, 121. [CrossRef]

73. Kozich, J.J.; Westcott, S.L.; Baxter, N.T.; Highlander, S.K.; Schloss, P.D. Development of a Dual-Index Sequencing Strategy and Curation Pipeline for Analyzing Amplicon Sequence Data on the MiSeq Illumina Sequencing Platform. Appl. Environ. Microbiol. 2013, 79, 5112-5120. [CrossRef] 
74. Sun, X.; Liu, D.; Zhang, X.; Li, W.; Liu, H.; Hong, W.; Jiang, C.; Guan, N.; Ma, C.; Zeng, H.; et al. SLAF-seq: An Efficient Method of Large-Scale De Novo SNP Discovery and Genotyping Using High-Throughput Sequencing. PLoS ONE 2013, 8, e58700. [CrossRef]

75. McKenna, A.; Hanna, M.; Banks, E.; Sivachenko, A.; Cibulskis, K.; Kernytsky, A.; Garimella, K.; Altshuler, D.; Gabriel, S.; Daly, M.; et al. The Genome Analysis Toolkit: A MapReduce framework for analyzing next-generation DNA sequencing data. Genome Res. 2010, 20, 1297-1303. [CrossRef] [PubMed]

76. Li, H.; Handsaker, B.; Wysoker, A.; Fennell, T.; Ruan, J.; Homer, N.; Marth, G.; Abecasis, G.; Durbin, R.; P. Genome Project Data. The Sequence Alignment/Map format and SAMtools. Bioinformatics 2009, 25, 2078-2079. [CrossRef] [PubMed]

77. Tamura, K.; Stecher, G.; Peterson, D.; Filipski, A.; Kumar, S. MEGA6, Molecular Evolutionary Genetics Analysis Version 6. Mol. Biol. Evol. 2013, 30, 2725-2729. [CrossRef] [PubMed]

78. Price, A.L.; Patterson, N.J.; Plenge, R.M.; Weinblatt, M.E.; Shadick, N.A.; Reich, D. Principal components analysis corrects for stratification in genome-wide association studies. Nat. Genet. 2006, 38, 904-909. [CrossRef]

79. Alexander, D.H.; Novembre, J.; Lange, K. Fast model-based estimation of ancestry in unrelated individuals. Genome Res. 2009, 19, 1655-1664. [CrossRef]

80. Hardy, O.J.; Vekemans, X. SPAGEDi: A versatile computer program to analyse spatial genetic structure at the individual or population levels. Mol. Ecol. Notes 2002, 2, 618-620. [CrossRef]

81. Barrett, J.C.; Fry, B.; Maller, J.; Daly, M.J. Haploview: Analysis and visualization of LD and haplotype maps. Bioinformatics 2005, 21, 263-265. [CrossRef]

82. Bradbury, P.J.; Zhang, Z.; Kroon, D.E.; Casstevens, T.M.; Ramdoss, Y.; Buckler, E.S. TASSEL: Software for association mapping of complex traits in diverse samples. Bioinformatics 2007, 23, 2633-2635. [CrossRef]

83. Lippert, C.; Listgarten, J.; Liu, Y.; Kadie, C.M.; Davidson, R.I.; Heckerman, D. FaST linear mixed models for genome-wide association studies. Nat. Methods 2011, 8, 833-835. [CrossRef]

84. Kang, H.M.; Sul, J.H.; Service, S.K.; Zaitlen, N.A.; Kong, S.Y.; Freimer, N.B.; Sabatti, C.; Eskin, E. Variance component model to account for sample structure in genome-wide association studies. Nat. Genet. 2010, 42, 348. [CrossRef]

85. Wang, P.; Lu, Q.; Ai, Y.; Wang, Y.; Li, T.; Wu, L.; Liu, J.; Cheng, Q.; Sun, L.; Shen, H. Candidate Gene Selection for Cytoplasmic Male Sterility in Pepper (Capsicum annuum L.) through Whole Mitochondrial Genome Sequencing. Int. J. Mol. Sci. 2019, 20, 578. [CrossRef] [PubMed]

(C) 2019 by the authors. Licensee MDPI, Basel, Switzerland. This article is an open access article distributed under the terms and conditions of the Creative Commons Attribution (CC BY) license (http://creativecommons.org/licenses/by/4.0/). 